\title{
Nanotechnology applied to the treatment of malaria ${ }^{2}$
}

\author{
Nereide Stela Santos-Magalhães ${ }^{a, *}$, Vanessa Carla Furtado Mosqueira ${ }^{\text {b }}$ \\ a Laboratório de Imunopatologia Keizo-Asami (LIKA), Universidade Federal de Pernambuco (UFPE), Recife, PE, Brazil \\ ${ }^{\mathrm{b}}$ Laboratório de Desenvolvimento Galênico e Nanotecnologia, Departmento de Farmácia, Universidade Federal de Ouro Preto (UFOP), 35400-000, Ouro Preto, Minas Gerais, Brazil
}

\section{A R T I C L E I N F O}

\section{Article history:}

Accepted 14 September 2009

Available online 13 November 2009

\section{Keywords:}

Malaria

Nanocarriers

Liposomes

Nanocapsules

Nanoparticles

Antimalarials

\begin{abstract}
A B S T R A C T
Despite the fact that we live in an era of advanced technology and innovation, infectious diseases, like malaria, continue to be one of the greatest health challenges worldwide. The main drawbacks of conventional malaria chemotherapy are the development of multiple drug resistance and the non-specific targeting to intracellular parasites, resulting in high dose requirements and subsequent intolerable toxicity. Nanosized carriers have been receiving special attention with the aim of minimizing the side effects of drug therapy, such as poor bioavailability and the selectivity of drugs. Several nanosized delivery systems have already proved their effectiveness in animal models for the treatment and prophylaxis of malaria. A number of strategies to deliver antimalarials using nanocarriers and the mechanisms that facilitate their targeting to Plasmodium spp.-infected cells are discussed in this review. Taking into account the peculiarities of malaria parasites, the focus is placed particularly on lipid-based (e.g., liposomes, solid lipid nanoparticles and nano and microemulsions) and polymer-based nanocarriers (nanocapsules and nanospheres). This review emphasizes the main requirements for developing new nanotechnology-based carriers as a promising choice in malaria treatment, especially in the case of severe cerebral malaria.
\end{abstract}

(c) 2009 Elsevier B.V. All rights reserved.

\section{Contents}

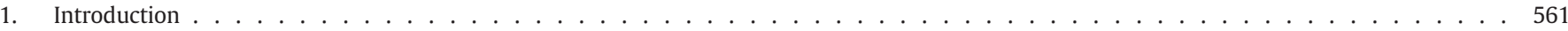

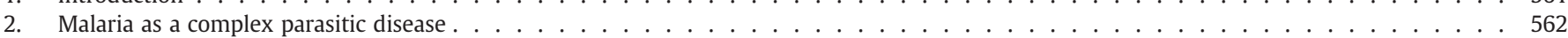

3. Current chemotherapy and drug-targeting approaches in malaria therapy . . . . . . . . . . . . . . . . . . . . . . . . . . . . . . . . . . . . . . . .

4. Nanotechnological strategies for drug targeting in malaria therapy . . . . . . . . . . . . . . . . . . . . . . . . . . . . 566

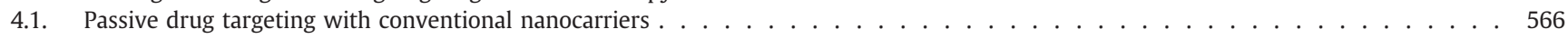

4.2. Passive drug targeting with hydrophilic surface-modified nanocarries . . . . . . . . . . . . . . . . . . . . . . . . . . 567

4.3. Active drug targeting with surface-modified nanocarriers . . . . . . . . . . . . . . . . . . . . . . . . . . . . . . . . 567

Abbreviations: ACT, artimisinin-based combination therapy; AE1, anion transport protein; AM, artemether; AS, artesunate; AT, atovaquone; AUC, area under the curve; CD,

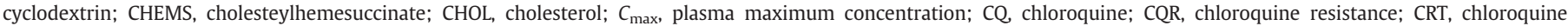

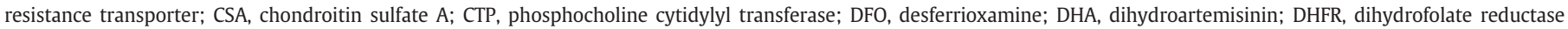

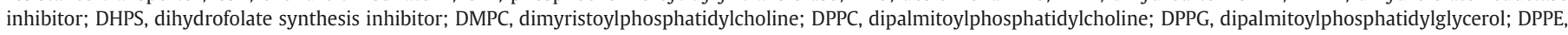

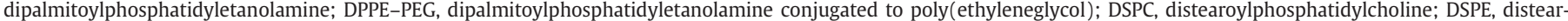

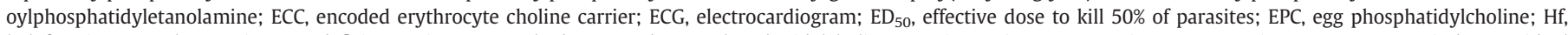

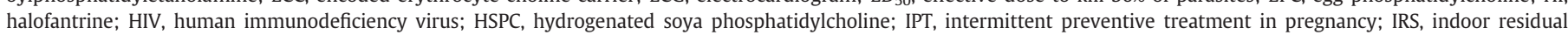

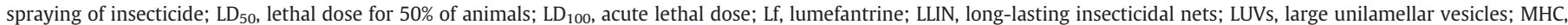

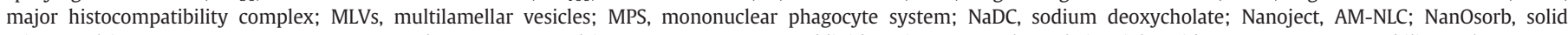

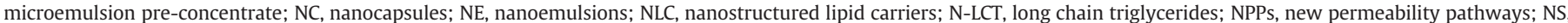

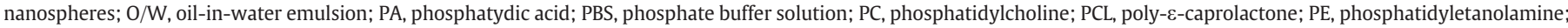

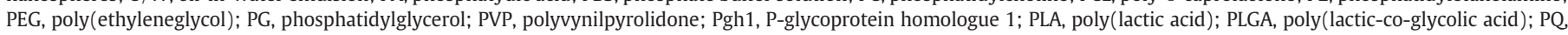

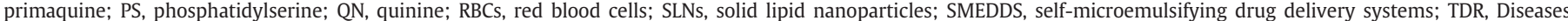
Research Programme of the World Health Organization; Tf, Transferrin; TQ Tafenoquine; WHO, World Health Organization; W/O/W, water-in-oil-in-water emulsion.

is This review is part of the Advanced Drug Delivery Reviews theme issue on "Nanotechnology Solutions for Infectious Diseases in Developing Nations".

* Corresponding author. Universidade Federal de Pernambuco (UFPE), Grupo de Sistemas de Liberação Controlada de Medicamentos, Laboratório de Imunopatologia Keizo-Asami

(LIKA), Av. Prof. Moraes Rego, 1235, Cidade Universitária, 50670-901, Recife, PE, Brazil. Tel.: +55 8121012501 ; fax: +55 81 21012508.

E-mail address: nssm@ufpe.br (N.S. Santos-Magalhães). 
5. Lipid-based nanocarriers for antimalarials and vaccines. . . . . . . . . . . . . . . . . . . . . . . . . . . . . . . . . . . 567

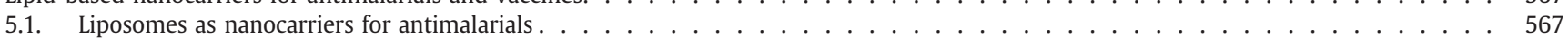

5.1.1. Conventional and long-circulating neutral liposomes . . . . . . . . . . . . . . . . . . . . . . . . . . . . . . . . . 568

5.1.2. Conventional and long-circulating negatively-charged liposomes. . . . . . . . . . . . . . . . . . . . . . . . . . . 568

5.1.3. Targeted liposomes for antimalarials. . . . . . . . . . . . . . . . . . . . . . . . . . . . . . . 569

5.2. Liposomes as adjuvants for malaria vaccines . . . . . . . . . . . . . . . . . . . . . . . . . . . . . . . . . . . 570

5.3. Solid lipid nanoparticles as nanocarriers for antimalarials . . . . . . . . . . . . . . . . . . . . . . . . . . . . . . . . . . . . 570

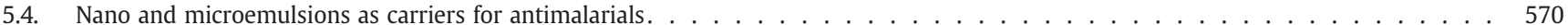

6. Polymeric-based nanocarriers for antimalarials. . . . . . . . . . . . . . . . . . . . . . . . . . . . . . . . . . . . . . . . . . 571

6.1. Polymeric nanoparticles as nanocarriers for antimalarials . . . . . . . . . . . . . . . . . . . . . . . . . . . . . . . . . . 571 .

6.2. Dendrimers as nanocarriers for antimalarials . . . . . . . . . . . . . . . . . . . . . . . . . . . . . . . . . . . . . 571

7. Other antimalarial nanocarriers . . . . . . . . . . . . . . . . . . . . . . . . . . . . . . . . . . 571

7.1. Cyclodextrins and inclusion complexes with antimalarials . . . . . . . . . . . . . . . . . . . . . . . . . . . . . . . . . 571

7.2. Nanosuspensions as carriers for antimalarials . . . . . . . . . . . . . . . . . . . . . . . . . . . . . . . . . . . . . . . . . 572

8. Nanocapsules: promising polymeric-lipid nanocarriers . . . . . . . . . . . . . . . . . . . . . . . . . . . . . . . . . 572

9. Conclusions . . . . . . . . . . . . . . . . . . . . . . . . . . . . . . . . . . . . . . . . 573

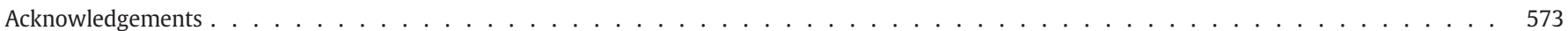

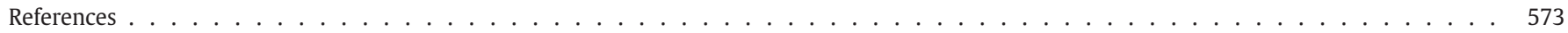

\section{Introduction}

Malaria, the most prevalent parasitic disease in the world, is caused by the apicomplex protozoan of the Plasmodium genus. Malaria is present all over the tropics, where four species, Plasmodium falciparum, Plasmodium vivax, Plasmodium malariae and Plasmodium ovale are transmitted to humans by the bites of the female mosquito vector of the Anopheles genus. In 2008, 109 countries were endemic for malaria, 45 of which are in Africa [1]. There were an estimated 247 million malaria episodes in 2006 . Around $86 \%$ of these cases occurred in Africa, causing over 1.25 million deaths [2]. The reason for such high morbidity and mortality stems from the fact that (i) the majority of the infections in Africa are caused by P. falciparum, the most dangerous of the four malarial parasites that infect humans, and (ii) the most effective malaria vector and the most difficult to control (Anopheles gambiae) is widespread in that continent. Approximately $80 \%$ of malaria cases in Africa were in 13 countries, and over half of them occurred in Nigeria, The Democratic Republic of the Congo, Ethiopia, Tanzania, and Kenya [3]. Among the cases that occurred outside Africa, 80\% occurred in India, Myanmar, Bangladesh, Indonesia, Papua New Guinea and Pakistan [1]. After Africa, India and Brazil are currently the regions of highest malaria endemicity in the world, being affected also by other Plasmodium species. Despite causing less mortality than $P$. falciparum, $P$. vivax is a widely distributed infection that has an enormous socioeconomic impact, being prevalent in South America, Asia and Oceania. There were approximately 881,000 malaria deaths in 2006 , of which $91 \%$ were in Africa $[1,2,4,5]$ and $85 \%$ were of children under 5 years of age. The majority of children experienced their first malaria infection during the first few years of life, when they have not yet acquired adequate clinical immunity [1]. For all, the efforts of the several not-for-profit partnership initiatives in the world, along with The Tropical Diseases Research Programme of the World Health Organization (TDR/WHO), malaria can still be considered a neglected disease because it suffers from insufficient research and development in therapy and vaccines worldwide, costing millions of lives. As shown in Fig. 1, South-East Asia and the African regions are the most affected areas in the world, with a combined population of more than 700 million. Currently, eighty countries are in the phase of malaria control; twelve countries are making the transition to an elimination programme; eleven countries are operating a malaria elimination programme, and six countries are actively engaged in preventing the reintroduction of malaria (Fig. 2). The latter are located along the edges of the global malaria distribution map (Fig. 1) [6].

Even though chemotherapy has been successful to some extent, failures are frequent and due to a variety of factors [7]. First, the intrinsic characteristics of the disease, related to the conditions of transmission and the difficult control of spreading through tropical areas $[1,8]$. Primary factors are the complexity of the parasite life cycle and the development of drug resistance $[2,6,9,10]$. Another critical factor is the increasing number of immune-compromised patients that suffer from malaria and human immunodeficiency virus (HIV) co-infections [11]. Reports suggest that the anti-malaria treatment failure is more common in HIV-infected adults with low CD4-cell counts than in HIV-uninfected patients. On the other hand, acute malaria episodes cause a temporary increase in the viral replication of HIV and consequently of the plasma viral load [12]. One study showed that HIV-infected children with advanced immune-suppression have more episodes of clinical malaria and higher parasite densities than HIV-infected children without advanced immune-suppression [13]. Another study showed a clear tendency to increased mortality in children with co-infection [14]. Together, these infections cause more than 4 million deaths a year.

Furthermore, the complexity of the recommended regimens, which are usually based on a combination of two or more drugs, increases the cost and reduces patient compliance, due to the severe side effects found (Table 1). In addition, extrinsic factors such as technical and operational failures in implementing campaigns to fight malaria, the poor quality of medicines distributed in different countries, drug interactions, the unavailability of less toxic drugs, resistance of the vector to insecticides and socioeconomic conditions of affected populations aggravate the difficulties for the eradication of malaria in the world [7].

The combination of tools and methods to arrest the widespread dissemination of malaria includes measures for the prevention of infection through the elimination of the mosquito using long-lasting insecticidal nets (LLIN) and indoor residual spraying of insecticide (IRS), and treatment with artemisinin-based combination therapy (ACT). As a last resort, intermittent preventive treatment with antimalarial drugs in the case of pregnancy (IPT) has been used to reduce the impact of malaria infection on the foetus during pregnancy [1]. Despite the fact that the most cost-effective measure for reducing the intolerable global burden of malaria would be the vaccination of the endemic population, an effective vaccine to control malaria is still not commercially available [15]. However, new vaccines that prevent malaria infection have been recently reported [16]. These vaccines, known as RTS,S/AS01B and RTS,S/ ASO2B, are undergoing phase I and II clinical trials and contain liposome-based and water-in-oil-based emulsion adjuvants, respectively. Both systems contain the immunostimulants monophosporyl lipid A and QS21, a triterpene glycoside purified from the bark of Quillaja saponaria [17]. 


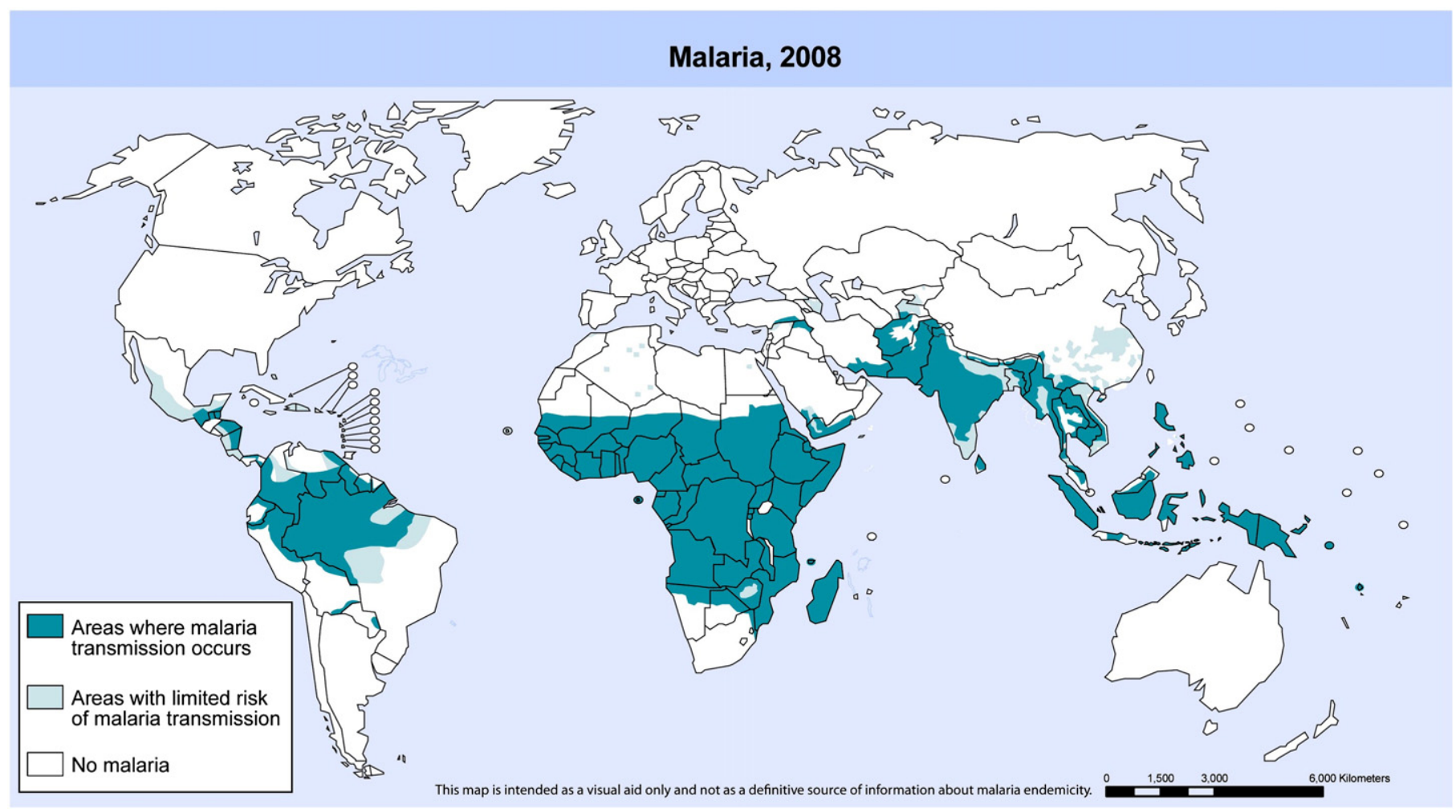

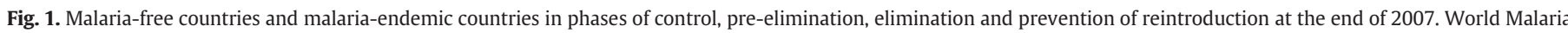
Risk Areas.

Source WHO 2008: [151].

In this setting, the rational use of drugs in combating malaria is crucial for reducing malaria disease and associated deaths. The treatment regimens of malaria are directly correlated to parasite drug resistance and dictated by government political strategies of prevention and control of morbidity and mortality due to the disease [18]. Considering the small number of new drugs or innovative antimalarial medicines approved since 1990, the search for more efficient and less toxic antimalarials, the development of a successful vaccine, and the design of nanotechnology-based delivery systems applied to drugs and antigens are likely to be the main strategies in combating this disease.

Nanocarriers are useful tools to improve the pharmacokinetic profile of effective drugs that due to poor water solubility, low bioavailability and high toxicity have been limitedly implemented in the pharmacotherapy [19-21]. Nanocarriers have been proposed for malaria diagnosis

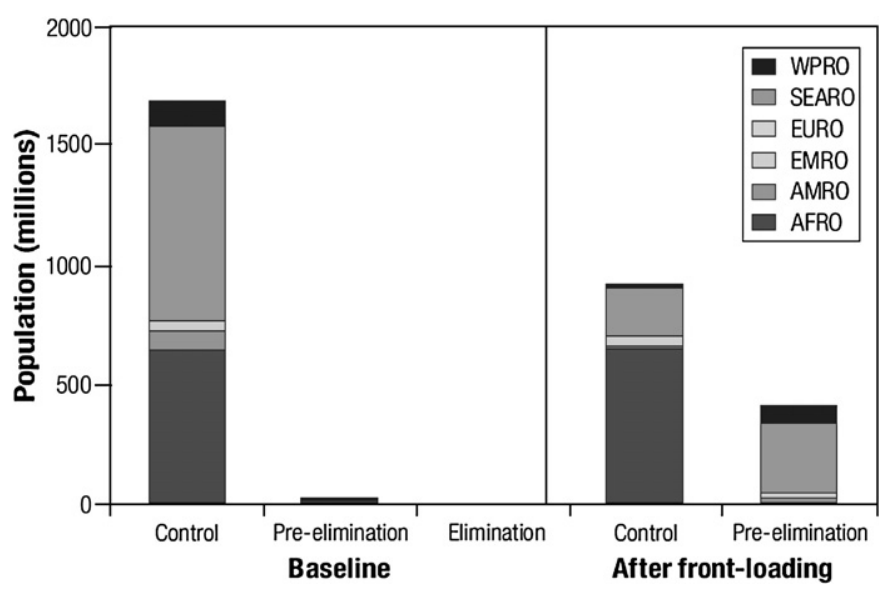

Fig. 2. Estimated world population in different phases of malaria control and elimination grouped by WHO regions.

Source: WHO 2008: [6].
[22,23], treatment $[21,24,25]$ and vaccine formulation [17,26-29]. The development of drug resistance by malaria parasites may also be due to the use of ineffective pharmaceutical dosage forms of antimalarials. Nanotechnology systems may therefore afford a better therapeutic outcome by targeting drugs specifically to their site of action. Malaria parasites frequently develop drug resistance due to the administration of low drug concentrations in the presence of a high parasitic count [30]. Furthermore, nanotechnology has the potential to restore the use of old and toxic drugs by modifying their biodistribution and reducing toxicity [21]. This advantage is particularly important in malaria therapy, since the development of new dosage forms for delivering drugs to parasiteinfected cells is urgently needed, especially for the antimalarials in clinical use. Nanocarriers may not only allow the use of potentially toxic antimalarials [25,31-33], but also increase the efficacy of the immune response in vaccine formulations [17,26,29,34].

The present review is intended to shed light on some biological aspects of malaria and to relate them to the use of nanotechnology as a promising strategy for malaria treatment. A number of approaches to deliver antimalarial agents and the mechanisms that facilitate their targeted delivery to Plasmodium-infected cells will be emphasized, taking into account the peculiarities of malaria parasites. The focus will be particularly on lipid-based nanocarriers such as liposomes, solid lipid nanoparticles, nanoemulsions and microemulsions, and on polymericbased nanosystems, such as nanocapsules and nanospheres for the treatment of malaria.

\section{Malaria as a complex parasitic disease}

In order to discuss the challenges associated with malaria therapy, the complexity of the disease should be taken into consideration and biological aspects of the parasite at different stages of its development in infected humans need to be understood. P. falciparum and P. vivax are the most prevalent Plasmodium species causing malaria. P. falciparum is the etiological agent of severe and potentially fatal malaria. P. vivax and 
Table 1

Conventional antimalarial drugs, cellular targets, advantages and disadvantages, clinical indications and drug-susceptibility of Plasmodium species. Source: $[4,5,7,36]$.

\begin{tabular}{|c|c|c|c|c|}
\hline Drug & Cellular targets & Advantages & Disadvantages & $\begin{array}{l}\text { Clinical indications/drug-susceptibility of Plasmodium } \\
\text { species }\end{array}$ \\
\hline \multirow[t]{3}{*}{$\begin{array}{l}\text { Chloroquine (CQ) phosphate, } \\
\text { hydroxychlroquine }\end{array}$} & Heme metabolism & $\begin{array}{l}\text { Fast action in RBC stages } \\
\text { Hydrophilicity, good bioavailability, high volume } \\
\text { of distribution }\end{array}$ & Widespread CQ-resistance, macular retinopathy & Uncomplicated malaria \\
\hline & Lysosomotropic & Oral dosage forms, low toxicity & $\begin{array}{l}\text { Very long terminal elimination half-time } \\
\text { (1-2 months) }\end{array}$ & P. falciparum (CQ-sensitive) \\
\hline & & & & $\begin{array}{l}\text { P.vivax (CQ-sensitive) } \\
\text { P. malariae }\end{array}$ \\
\hline \multirow[t]{4}{*}{ Quinine $(Q N)$ sulfate } & Heme metabolism & Fast action in RBC stages, hydrophilic drug & Less potent than CQ & Severe malaria (IM/IV) \\
\hline & & Oral route formulation & Drug association is needed & P. vivax and P. falciparum \\
\hline & & High oral bioavailability resistance is uncommon & Small therapeutic index, bolus i.v. is forbidden & CQ-resistant uncomplicated malaria \\
\hline & & & $\begin{array}{l}\text { Life-threatening adverse effects } \\
\text { Cinchonism, hypoglycaemia }\end{array}$ & Association with doxycycline, tetracycline, or clindamycin \\
\hline Artemisinin: & $\begin{array}{l}\text { Inhibits PfATP6 outside the } \\
\text { parasite's food vacuole }\end{array}$ & Safe and well-tolerated & Neurotoxicity dose-dependent, & Severe complicated malaria management \\
\hline Dihydroartemisinin & & Potent and fast action in blood stages & Very short-elimination half-lives, & P. falciparum CQ-resistant \\
\hline Artesunate (AS) & & Gametocytocidal effects & Low bioavailability by oral route & \\
\hline Artemether (AM) & & No widespread resistance & $\begin{array}{l}\text { Poor water solubility } \\
\text { Unavailable i.v. dosage form }\end{array}$ & \\
\hline \multirow{2}{*}{ AM and Lumefantrine (Lf) } & Many targets protein metabolism & Well-tolerated & Expensive drug & Uncomplicated malaria \\
\hline & & & Lf: elimination half-life of $1-6$ days & Multi-resistant $P$. falciparum malaria \\
\hline \multirow[t]{2}{*}{ Mefloquine (MQ) } & Heme metabolism & Potent action in RBC stages, & $\begin{array}{l}\text { Higher rate of severe neuropsychiatric reactions } \\
\text { Very long half-life }\end{array}$ & Uncomplicated malaria \\
\hline & & P. vivax (gametocidal) & Serious side effects, rather expensive & $\begin{array}{l}\text { Multi-resistant } P \text {. falciparum malaria } \\
\text { P. vivax CQ-resistant } \\
\text { P. malariae }\end{array}$ \\
\hline \multirow[t]{2}{*}{ MQ and AS } & & Clear parasitemia in shorter time periods & Single AS is unable to cure infected patients & Uncomplicated malaria \\
\hline & & & $\begin{array}{l}\text { Very slowly eliminated (15-33 days) } \\
\text { Stillbirth risk during pregnancy }\end{array}$ & Drug-related resistance (P. falciparum) \\
\hline \multirow[t]{3}{*}{ Halofantrine (Hf) } & Heme metabolism & Fast action in RBC stages & Incomplete and variable absorption & Uncomplicated malaria \\
\hline & & 1-2 days terminal half-life elimination & High cost & P. falciparum (CQ-resistant and CQ-multi-resistant ) \\
\hline & & & $\begin{array}{l}\text { Serious side effects: QT prolongation/ECG } \\
\text { arrhythmmia }\end{array}$ & \\
\hline \multirow[t]{2}{*}{ Amodiaquine $(\mathrm{AQ})$} & Heme metabolism & Gametocidal, & $\begin{array}{l}\text { Widespread across-resistance with } \mathrm{CQ}(P . \\
\text { falciparum })\end{array}$ & Uncomplicated malaria \\
\hline & & Good tolerability & Causes hepatitis and agranulocytosis (chronic use) & $\begin{array}{l}\text { P. vivax } \\
\text { P. malariae }\end{array}$ \\
\hline \multirow[t]{2}{*}{ Atovaquone (AT) } & AT inhibits mitochondrial respiration & & Poorly and variably absorbed & Uncomplicated malaria \\
\hline & & & Long elimination half-life $(50-70 \mathrm{~h})$ & $\begin{array}{l}\text { P. falciparum (CQ-resistant) multi-resistant malaria } \\
\text { chemoprophylaxis }\end{array}$ \\
\hline Proguanil (PG) & & & Very expensive drug & \\
\hline Pyrimethamine & Dihydrofolate reductase inhibitor & $\begin{array}{l}\text { Proguanil and chlorproguanil: } \\
\text { short-elimination half-lives }\end{array}$ & Metabolism varies considerably & Uncomplicated malaria \\
\hline $\begin{array}{l}\text { Biguanides: proguanil and } \\
\text { chlorproguanil }\end{array}$ & (DHFR) & Oral dosage forms & $\begin{array}{l}\text { Antimalarial activity due to triazine metabolites } \\
\text { (cycloguanil, chlorcycloguanil) }\end{array}$ & P. falciparum (CQ-resistant) \\
\hline chlorproguanil-dapsone & Folate inhibitors & & & $\begin{array}{l}\text { Uncomplicated malaria } \\
\text { P. falciparum (CQ-resistant) }\end{array}$ \\
\hline Sulfadoxine-pyrimethamine & $\begin{array}{l}\text { DHFR- and DHPS-inhibitor } \\
\text { combination }\end{array}$ & $\begin{array}{l}\text { Pyrimethamine: well absorbed after } \\
\text { oral or i.m. administration }\end{array}$ & Resistance, idiosyncratic toxicity & Uncomplicated malaria \\
\hline \multirow[t]{2}{*}{ Pyrimethamine-dapsone } & & & Sulfadoxine: long elimination half-life (100-200 h) & Prophylaxis \\
\hline & & & Sulfadoxine: severe allergic reaction & P. falciparum (CQ-resistant) \\
\hline \multirow[t]{2}{*}{ Primaquine $(\mathrm{PQ})$ phosphate } & Interference with ubiquinone & Prophylactic action & $\begin{array}{l}\text { Hemolytic anemia, methemoglobinemia and } \\
\text { hemeolysis in patients with G6PD deficiency }\end{array}$ & Prophylaxis: hypnozoite stages \\
\hline & Unclear function in mitochondria & Active against hypnozoites & $\begin{array}{l}\text { Must not be used during pregnancy } \\
\text { Limited oral availability }\end{array}$ & P. vivax and $P$. ovale (radical cure) \\
\hline
\end{tabular}


P. ovale have dormant liver-stage parasites (hypnozoites). P. malariae produces long-lasting infections and, if untreated, can persist asymptomatically in the human host for years, even a lifetime [8]. The life cycle of malaria in a human host starts when the infected mosquito bites the individual (Fig. 3A). The inoculated sporozoites migrate to the liver and invade the hepatocytes by mechanisms still not entirely understood [35]. A sporozoite generates about 30,000 new parasites in the case of P. falciparum or 10,000 in P. vivax infection. P. vivax induces slowdeveloping sporozoites in liver cells, known as hypnozoites, which remain for months in this tissue. Hypnozoites induce new relapses of the disease up to two years after the initial infection. Meanwhile, the asexual blood stages (late trophozoites and schizonts) of the parasite are the main etiological agents in malaria, causing the symptoms of the disease to appear about two weeks after the mosquito-vectored sporozoite inoculation (Fig. 3A) [35]. These asexual intra-erythrocytic stages are the main target of the antimalarial drugs [7,9]. Invasive forms, known as merozoites, enter the red blood cells $(\mathrm{RBC})$ and remain relatively inactive metabolically for $10-15 \mathrm{~h}$ (the ring stage) (Fig. 3A). The parasite then undergoes a rapid phase of growth over the next $25 \mathrm{~h}$, constituting the trophozoite stage; the parasite digests the majority of the hemeoglobin and grows to fill $>50 \%$ of the volume of the host cells (Fig. 3B). Hemeoglobin is digested within a food vacuole (Fig. 3B), which results in the formation of heme. Once the heme has been formed, it associates via one of the peripheral carboxyl groups with the $\mathrm{Fe}^{3+}$ of an adjacent heme to produce insoluble hemeozoin. At the end of the trophozoite stage, the parasite divides several times (the schizont stage) before the host cell lyses (some $48 \mathrm{~h}$ after invasion) to release the newlyformed merozoites that continue the cycle. This concerted cell lysis triggers the classical malaria symptoms, which are mainly headache, periodically recurrent high fever (every 48-72 h), myalgia, anemia, hepatitis and splenomegaly $[9,36]$. Furthermore, neurological symptoms occur and may result in death in the case of severe P. falciparum infection. This severe form of the disease, known as cerebral malaria syndrome, may be an important cause of neurological handicap in childhood in the tropics [37]. The pathogenesis of cerebral malaria is multifactorial, being caused by parasite sequestration and blockage of blood flow in small vessels of the brain, resulting in clogging, sequestration, rosette formation, release of cytokines, cerebral oedema and increased intracranial hypertension [38,39]. If treatment is not promptly instituted, the disease caused by P. falciparum can lead to death, especially in acute primary infections [40].

\section{Current chemotherapy and drug-targeting approaches in malaria therapy}

Chemotherapy is the strategy to reduce the malaria-associated morbidity and mortality. The decision leading to the appropriate treatment for the malaria-infected patient must be preceded by the

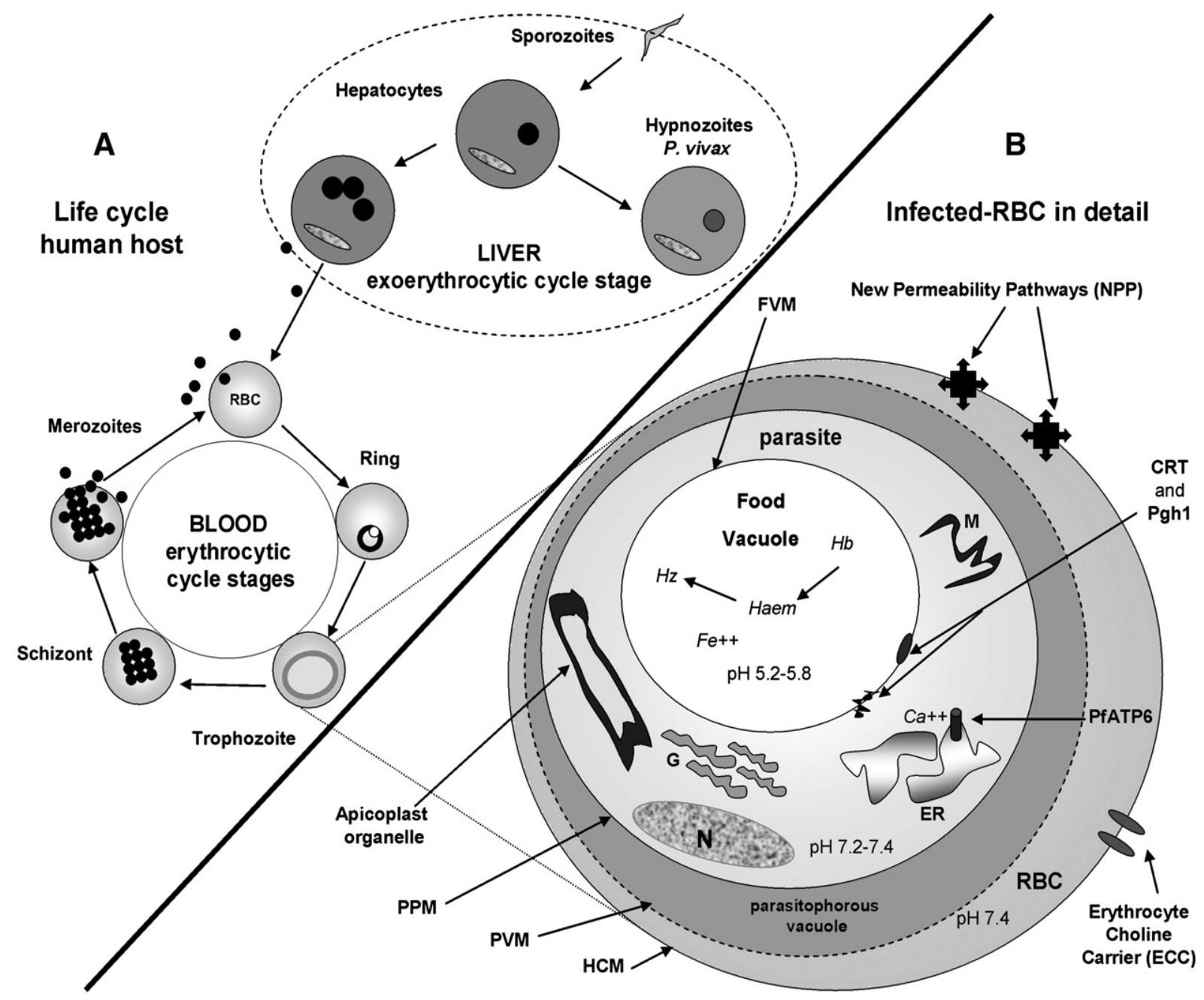

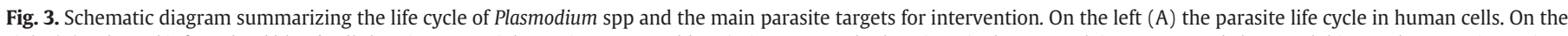

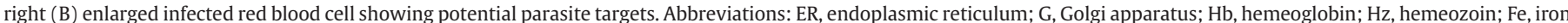

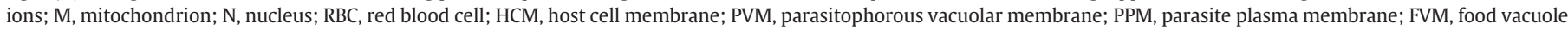

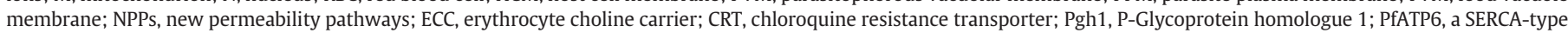
$\mathrm{Ca}^{2+}$ ATPase in endoplasmic reticulum proposed to be one of the target of artemisinins. Adapted and redrawn from [9,43-45,48,56,57]. 
identification of the infecting Plasmodium species, definition of the seriousness of the disease, the existence of pre-infection exposure, the determination of the clinical status of the patient and drug susceptibility, as determined by the geographic area where the infection was acquired [7]. Table 1 summarizes the drugs or drug associations used in malaria treatment caused by different Plasmodium species and clinical indications, as well as the main advantages and disadvantages of each drug. Currently, a single effective treatment for malaria caused by the most prevalent species of Plasmodium ( $P$. falciparum and $P$. vivax) is not possible, due to the differences in Plasmodium species susceptibility to the recommended drugs $[7,41,42]$.

Chloroquine (CQ) has several pharmacokinetic and pharmacological advantages over all the other antimalarial drugs, which accounts for its excellent performance over eight decades of malaria therapy, despite the CQ-resistance developed by Plasmodium strains. The mode of action of CQ is based on its accumulation in the food vacuole of the parasites (lysosomotropic character). This interferes with the polymerization of the toxic heme monomers [7,43-45]. CQ leads to parasite death by heme poisoning. The main advantages of CQ therapy are the fast action in blood parasite stages, low toxicity, good bioavailability from oral dosage form, water solubility, high volume of distribution in the body and low cost (Table 1). Primaquine (PQ) is a schizontocide drug used in the prophylaxis against all types of malaria, preventing the primary installation of parasitemia. PQ is the only commercially available drug active on presumptive anti-relapse therapy, known as terminal prophylaxis and preventing late relapses of the disease caused by hypnozoites of $P$. vivax and $P$. ovale. PQ promotes a radical cure in individuals infected with $P$. vivax or P. ovale [46] and is also active on gametocytes (sexual erythrocytic forms) in a single dose [10]. However, $\mathrm{PQ}$ is a very toxic drug (Table 1 ).

More recently, tafenoquine (TQ), a PQ analogue, was tested clinically and is presently under development [47]. TQ is highly effective in both the radical cure of relapsing malaria and the prophylaxis of $P$. vivax and $P$. falciparum infections, revealing a protective efficacy of over $90 \%$ and a safe profile. Nowadays, TQ represents a potentially exciting alternative to PQ for preventing infection and for the radical cure of malaria [46,47].

As a result of their genetic diversity, the Plasmodium strains have demonstrated an almost peculiar ability to evade the unfavourable conditions imposed by drug therapy, and have developed complex mechanisms of drug resistance. Thus, the vast majority of widely used antimalarial therapies have lost their usefulness over time [41,42]. CQ and multi-drug resistance have emerged as the most important challenges facing malaria treatment $[41,42,45]$. Resistance is attributed to mutations on the $P$. falciparum chloroquine transporter gene (pfcrt) and the P. falciparum multi-drug resistance gene (pfmdr1), respectively [48]. The products of the gene expression are two putative protein transporters exhibited by P. falciparum, the CQresistance transporter (CRT) and the P-glycoprotein homologue 1 (Pgh1), which are implicated in the phenomenon of CQ-resistance (CQR). Both CRT and Pgh1 are present on the digestive vacuole membrane of Plasmodium (Fig. 3B). CQ-resistant Plasmodium strains accumulate fewer amounts of drug in the digestive vacuole and are, therefore, able to protect themselves against drug damage $[44,45,49]$. Under continued drug exposure, $P$. falciparum resistant strains become dominant over time. Pgh1 is also implicated in the resistance of $P$. falciparum to mefloquine (MQ), with amplification of the gene encoding Pgh1 associated with both in vitro and in vivo MQ resistance $[42,43]$.

To prevent drug-resistance mechanisms in malaria therapy, new strategies for intracellular antimalarial delivery are urgently needed [45]. Both CQ transporters could be probably bypassed by drugs encapsulated in nanosystems able to pass through the digestive vacuole membrane by different mechanisms [50,51]. These mechanisms could, for example, be membrane fusion and membrane-induced instability triggered by $\mathrm{pH}$ differences in the cell compartments. These approaches can be exploited in order to reverse CQ-resistance using nanocarriers loaded with CQ. One of these suggested strategies has already been reported with CQ entrapped in $\mathrm{pH}$-sensitive liposomes, even though in vivo investigations were not carried out [52]. Another possible approach is the use of nanocarriers containing antisense nucleotides that inhibit the expression of CQ-resistance phenotype of membrane transporters.

The route of administration is also an important aspect to be considered in malaria. The oral route should be the first choice for clinically uncomplicated malaria. However, parenteral therapy is advocated in severe or complicated malaria and intravenous administration is obligatory in the management of cerebral malaria. The mainstay of cerebral malaria therapy is the use of either quinine (QN) or artemisinin, both of which are effective antimalarials by the parenteral route. However, QN presents high toxicity when administered intravenously and artemisinin derivatives are lipophilic and present short plasma half-lives upon parenteral administration (Table 1) [53,54]. On the basis of these findings, there is an urgent need to develop intravenous efficacious formulations able to reduce the side effects of QN and improve the solubility and pharmacokinetic profile of artemisinin in the treatment of cerebral malaria.

The main goal of malaria therapy is to promote a high drug concentration in the intracellular parasitophorous vacuole where the Plasmodium is hosted $[9,55]$. When developing a new antimalarial therapy, it should be borne in mind that the parasite is inside RBCs (Fig. 3B) and there are multiple membranes that must be traversed by antimalarial drugs in order to access intraparasitic targets. These cell barriers comprise, in an orderly fashion, the host cell membrane ( $\mathrm{HCM})$, the parasitophorous vacuolar membrane (PVM), the parasite plasma membrane (PPM) and, depending on the site of action of the drug, an organelle membrane such as a food vacuole membrane (FVM) or an endoplasmic reticulum membrane (ERM) [48]. There are differences in the $\mathrm{pH}$ of these above-mentioned intracellular compartments in the Plasmodium-infected RBC (Fig. 3B) that could be exploited to selectively deliver antimalarial drugs using $\mathrm{pH}$-sensitive nanosystems. In this context, a number of schematic targets for new antimalarial drug candidates and drug-loaded nanocarriers preferentially affecting parasites with minimal toxicity for the host are shown in Fig. 3B.

First, it is well known that an increase in the permeability of the membranes of infected-RBCs to a wide range of low-molecular-weight solutes occurs following host infection by Plasmodium; membrane channels appear 12-16 h after Plasmodium invasion [48]. These channels are called 'new permeability pathways' (NPPs) and allow the differentiation between infected and non-infected RBCs (Fig. 3B). It was reported that macromolecules such as dextran, protein A and IgG2a antibody gained access to the parasite through NPPs in infected RBCs [56]. Moreover, using a variety of fluorescent latex beads, it was determined that beads up to $50-80 \mathrm{~nm}$ in diameter were able to access intracellular parasites in infected-RBCs [57]. By analogy, it may be hypothesized that antimalarial nanocarriers $(<80 \mathrm{~nm}$ ) could also access intracellular compartments of the parasite through NPPs in infected RBCs [58]. NPPs can thus be exploited as a target for antimalarial nanocarriers, as indicated in Fig. 3B.

On the other hand, phospholipid metabolism is absent in mature erythrocytes but is rampant in infected RBCs (the phospholipid content increases by $500 \%$ ) due to the de novo biosynthesis pathway of phosphatidylcholine (PC) in which choline is actively salvaged from the host serum [48]. Choline transport from serum to RBCs is affected by erythrocyte choline carriers (ECC). The parasite-encoded ECC involved in choline uptake by infected-RBCs offers a great potential for the selective targeting of potent antimalarial drugs (Fig. 3B). Moreover, the mechanism of inhibition of the de novo biosynthesis pathway of phosphatidylcholine (PC) in Plasmodium has been elucidated and it is considered as a new target for antimalarial drugs. In fact, choline analogues have been synthesized as pro-drugs named T3 and T4, and their respective bio-precursors, TE3 and TE4a. These compounds have presented outstanding antimalarial activity, as expressed by the 
effective dose to kill $50 \%$ of parasites $\left(E D_{50}=0.1-0.25 \mathrm{mg} / \mathrm{kg}\right)$, inhibiting the asexual blood stages of $P$. falciparum [59]. These drugs specifically accumulate inside infected RBCs, block phosphatidylcholine de novo biosynthesis and interact with hemozoin. Furthermore, T16, another PC de novo biosynthesis inhibitor, has displayed potent in vitro activity against $P$. falciparum at a nanomolar concentration (1.9 $\mathrm{nM})$, highlighting the unique ability of this class of compounds to selectively accumulate within and kill apicomplexa-infected RBCs [60]. This mode of action, different from that of current antimalarial drugs, is a major advantage of PC de novo biosynthesis inhibitors.

In addition, the apicoplast (Fig. 3B), an organelle ancestrally related to chloroplasts, has also emerged as a promising target for new selective antimalarial drugs $[48,61]$. This is a nonessential pathway in the asexual blood stages of Plasmodium, but is a key to the development of infectious liver-stage forms [45]. Summarizing, due to chemotherapy selectivity, the parasite-specific metabolic pathways are the most attractive targets [43]. To better understand these mechanisms of drug targeting, readers should consult a number of interesting currently available reviews [43-45,48,62].

\section{Nanotechnological strategies for drug targeting in malaria therapy}

The aim of using nanocarriers as drug delivery systems is to promote drug or vaccine protection against extracellular degradation, to improve selectivity in relation to the target, to reduce the frequency of administration and the duration of the treatment and to improve the pharmacokinetic profile of the drug $[20,63,64]$. For the purposes of this review, the terms "nanosystems" or "nanocarriers" include all the drug carrier systems displaying sizes $<1000 \mathrm{~nm}$. The design of new nanocarriers should consider that in chemotherapy the plasma maximum concentration $\left(C_{\max }\right)$ of a drug is proportional to its toxic effects and the efficacy is proportional to the area under the curve (AUC) of drug plasma concentration [65]. Nanoparticulate drug delivery systems represent a promising approach for obtaining desirable druglike properties by altering the biopharmaceutics and pharmacokinetics property of the drug molecule [64]. In general, long-circulating nanosystems are able to improve the AUC of the drugs and reduce the doses employed in chemotherapy, due to their enhanced selectivity [66].

The most important property of a nanocarrier in the context of malaria is the ability to remain in the blood stream for a long period of time in order to improve the interaction with infected red blood cells (RBCs) and parasite membranes [32]. Additional interesting properties are protection of instable drugs, cell-adhesion properties, and the ability to be surface-modified by conjugation of specific ligands [24,25]. It is noteworthy that, in the treatment of cerebral malaria, most of these potential benefits can be achieved by colloidal nanocarriers that fit intravenous administration. In uncomplicated malaria, the non-parenteral routes are preferred, but they reduce the spectrum of possibilities in terms of the use of drug nanocarriers. Many efforts have been made to meet the implement nanotechnologies in the context of malaria [32,6773]. The next sections will be devoted to discuss these attempts.

The main strategies for targeting antimalarial drugs to the infected erythrocytes and occasionally the hepatocytes using nanocarriers by the intravenous route are passive and active targeting. Passive targeting is achieved using conventional nanocarriers (e.g., liposomes, hydrophobic polymeric nanoparticles) [63], or surface-modified long-circulating nanocarriers (e.g., PEGylated) [20,74,75]. In contrast, active targeting is attained by means of nanocarriers surface-modified with specific ligands such as carbohydrates, proteins, peptides or antibodies [63]. These two approaches to the targeting of antimalarials are illustrated in Fig. 4 (A, B and C) and will be detailed in the following sections.

\subsection{Passive drug targeting with conventional nanocarriers}

Passive targeting refers to the accumulation of the drug-loaded carrier at a particular body site due to physicochemical or pharmacological
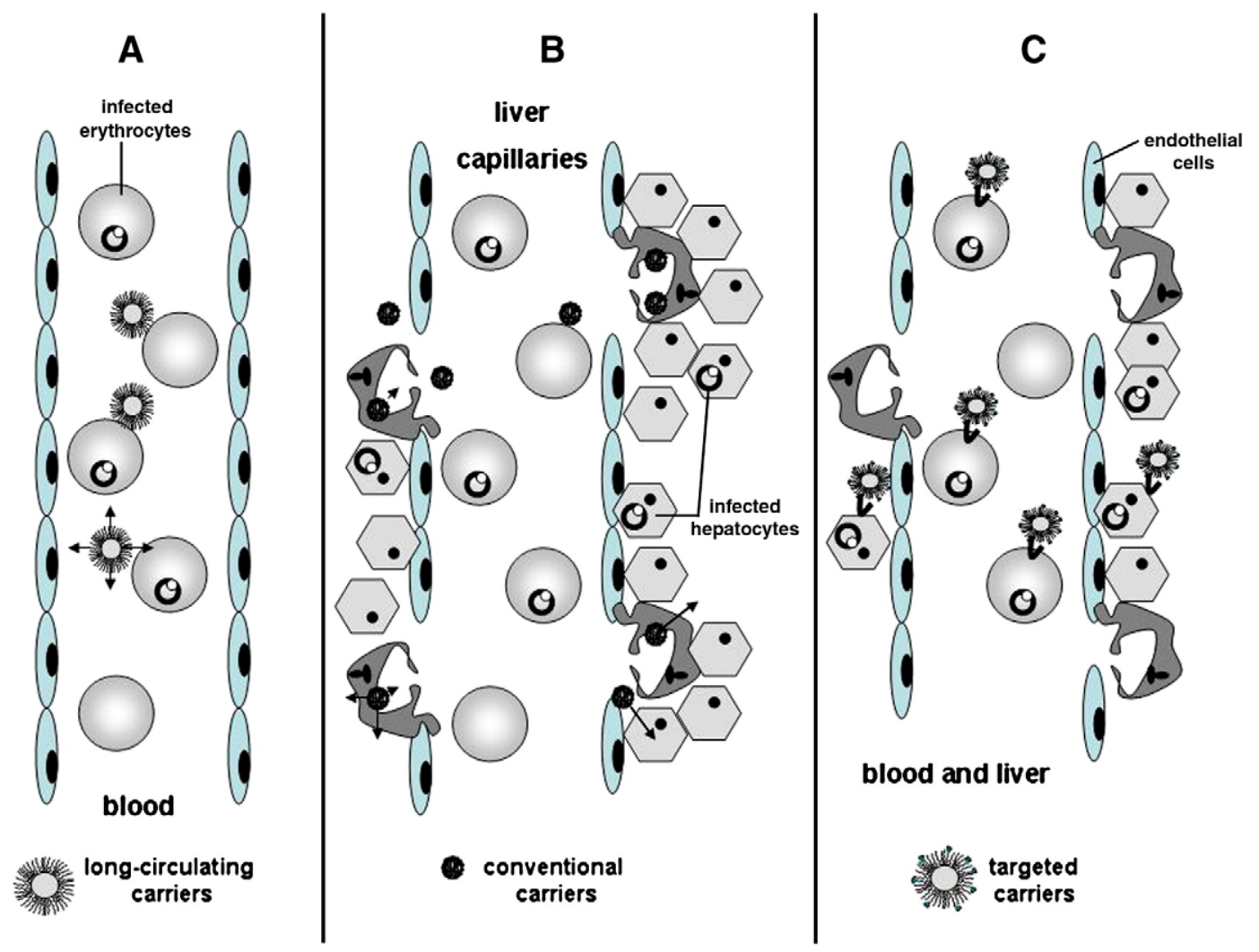

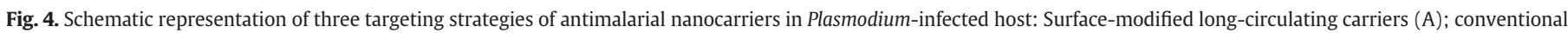
surface-unmodified carriers (B) and ligand-modified carriers to improve selective targeting to infected erythrocytes and hepatocytes (C). 
factors [63,76]; nanocarriers are passively targeted, making use of the pathophysiological and anatomic features [76]. Cells of the mononuclear phagocyte system (MPS) are a simple target due to their phagocytic properties. In contrast, cells that are deprived of phagocytic activity are much more difficult to target. In fact, passive targeting is less exploited in malaria than in leishmaniasis therapy by the intravenous route, because of the differences in the type of infected host cells (RBCs and MPS, respectively). RBCs are phagocytically and endocytically inactive. However, it should be emphasized that conventional nanocarriers employed by the parenteral route are rapidly taken up by MPS cells, delivering the drug inside macrophages. Thus, exposure of phagocytes to nanocarrier overload could lead to an initial blockage of the phagocytic uptake that is resolved within 24 to $48 \mathrm{~h}$, followed by a subsequent two-fold rise in the macrophage capacity [77]. This could reduce the rapid action of an antimalarial drug entrapped in a nanocarrier, but may be a very interesting strategy to generate a depot that releases slowly into the blood, thereby altering the pharmacokinetic profile of a short half-life antimalarial drug. On the other hand, the use of conventional nanocarriers for treating $P$. vivax infections in which hypnozoites are the dormant forms of the parasite in the hepatocytes, located side by side with Kupffer cells, may be an interesting strategy (Fig. 4B). For example, PQ was entrapped in conventional nanocapsules (NC) and nanospheres (NS) for macrophage targeting. These formulations were tested in vitro and in vivo against leishmaniasis, inducing toxicity reduction [78,79]. Unfortunately, experiments in a malaria model with PQ-loaded nanocarriers were not performed and further investigations are required to validate this approach.

\subsection{Passive drug targeting with hydrophilic surface-modified nanocarries}

The surface-modification of nanocarriers with hydrophilic polymers such as poly(ethyleneglycol) (PEG) delays phagocytosis, resulting in a prolonged drug half-life in the blood and allowing the modulation of the biodistribution and the pharmacokinetic profile of the drug [75,80,81]. Mechanisms include reduced protein adsorption and limited opsonization and complement activation [80,82].

Passive targeting to MPS can be used in malaria therapy, though longcirculating nanocarriers seem to be more suitable for the intravenous delivery due to the increased contact with RBCs (Fig. 4A). Also, the reduced volume of distribution of the antimalarial can potentially result in less toxic effects to the tissues [32,67]. One worth mentioning example is the halofantrine (Hf)-loaded-nanocapsules ( $\mathrm{NC}$ ); $\mathrm{Hf}$ is a very hydrophobic drug used in the treatment of malaria [83,84]. Two formulations were designed for intravenous administration: (i) conventional poly(lactic acid)-NC without surface modification and (ii) PLA-PEG NC. Despite the different biodistribution profiles of unloaded NC [83], only a slight difference between the pharmacokinetic parameters of $\mathrm{Hf}$ encapsulated in both formulations was found when they were evaluated in $P$. berghei-infected mice $[32,67]$. These unexpected results might be a consequence of performing the experiments with heavily-parasitized mice as opposed to healthy animals.

The involvement of the liver and spleen in clearing senescent and parasitized RBC in infected mice might saturate the MPS, thus affecting NC pharmacokinetics, reducing the difference between conventional PLA-NC and long-circulating PLA-PEG NC. This example clearly shows that passive targeting was probably achieved in malaria-infected mice even in those with unmodified PLA nanocapsules, because of MPS saturation. On the other hand, these results suggest that infected-mice models should be used to establish the real pharmacokinetic profile of an antimalarial associated with $\mathrm{NC}$, taking into account the influence of the disease on nanocarrier biodistribution [67].

\subsection{Active drug targeting with surface-modified nanocarriers}

Active targeting of therapeutic drugs associated with nanocarriers is achieved by conjugating a cell-specific ligand at the surface of the carrier, thereby allowing a preferential accumulation of the drug in the target cell or tissue [81]. This approach may be successful if the receptors for surface-bound ligands are expressed uniquely in diseased cells or if their expression is differentially higher in diseased cells as compared to normal ones. In the case of malaria, erythrocytes in the blood and hepatocytes in the liver are the main targets (Fig. 4C). The identification of new Plasmodium or infected cell targets can also be used to modify existing drug delivery systems employing nanotechnology to more efficiently deliver antimalarial drug molecules to the newly-targeted sites of action. However, the disadvantage in this approach is that ligand-attached nanocarriers may induce an undesirable immunological response, due to the proteic nature of some ligands. Another challenge is to adjust the number of ligands per nanocarrier and the suitable PEG chain length at the surface of nanocarriers to properly expose the ligand for cell recognition [81]. Even though this it is a difficult task, the benefits of such a strategy may have an important role in malaria treatment, especially for parenteral administration in the management of cerebral malaria. Thus, the targeting of moieties that are natural candidates for attachment to nanocarriers are peptides, antibodies and, particularly, small carbohydrate-based molecules.

Few attempts using active targeting strategy in experimental malaria treatment with colloidal nanocarriers have been investigated $[69,85]$. To date, the active targeting approach was explored using liposomes [86-91] and solid lipid nanoparticles (SLN) [71]. Strategies dealing with each type of nanocarrier will be discussed in the sections below.

\section{Lipid-based nanocarriers for antimalarials and vaccines}

\subsection{Liposomes as nanocarriers for antimalarials}

Liposomes are vesicles formed by an aqueous core surrounded by one or several phospholipid bilayers; hydrophilic drugs or active compounds can be incorporated into the inner aqueous cavity and lipophilic drugs imbibed into the phospholipid bilayer [74]. The pioneering work by Bangham in 1965, when studying biological membrane characteristics [92], demonstrated that phospholipids dispersed in water can be organized in multilamellar vesicles known as liposomes. Later, Gregoriadis introduced the concept of drug delivery systems using liposomes to carry enzymes, proteins and drugs [93,94].

Subsequently, the use of liposomes has been extended to encapsulate drugs [95-97] and biologically active compounds $[98,99]$ and to serve as immunological adjuvants in vaccination $[100,101]$. In the early 1980 s, a new strategy to avoid the uptake of liposomes by MPS was developed [74,102]. Later on, the surface of liposomes was modified with antibodies, peptides or other ligands to target drugs to specific sites [86-88,90]. For the detailed classification of liposomes and the evolution of their sophistication as drug delivery systems, readers are advised to refer to recently published reviews compiled by Torchilin $[81,103]$.

Although liposomes present numerous in vitro and in vivo stability challenges $[81,103]$, due to their versatile multifunctional properties, they remain the best choice as drug carriers by either passive or active targeting. The targeting of liposomes to MPS can be enhanced by the presence of grafted-surface ligands that not only bind specifically to the MPS cells, but also enhance their natural killer activity [90], as discussed above. In view of this, liposomes are suitable nanocarriers for the prophylaxis and treatment of malaria, carrying vaccines or antimalarials, respectively.

Conventional neutral multilamellar liposomes, prepared with egg phosphatidylcholine (EPC) and cholesterol (CHOL), were the first nanocarriers proposed for antimalarial drugs [104]. At that time, research on liposomal antimalarial systems emphasized the technological aspects of the design and in vitro and in vivo stability of liposomes to allow them to reach erythrocytes and MPS $[31,87,105]$. 
Subsequently, advances in liposome functionality, long-circulating and targeted liposomes have been proposed for carrying drugs and to develop vaccines to prevent malaria [71,87,90]. For example, conventional and long-circulating negatively-charged liposomes prepared with EPC, CHOL and phosphatidylglycerol (PG) or phosphatidylethanolamine (PE) conjugated to PEG were used to encapsulate antimalarials [52]. More recently, lyophilized liposomal formulations for encapsulating antimalarial drugs before use have been proposed [87]. In the following sections of this review, studies aimed at evaluating the influence of the surface charge of conventional, longcirculating and targeted liposomes on the encapsulation, release pattern and bioavailability of different antimalarials will be discussed.

\subsubsection{Conventional and long-circulating neutral liposomes}

Since the 1980s, antimalarial drugs such as QN, CQ PQ, artemether (AM) and artesunate (AS) have been encapsulated in neutral conventional or long-circulating liposomes using different preparation techniques. The findings have shown that the $\mathrm{pH}$ gradient technique seems to be the best for improving the encapsulation efficiency of antimalarials, especially in the case of quinolones. These molecules are positively charged in physiological $\mathrm{pH}$ and their encapsulation can therefore be controlled by a pH gradient.

To protect the drug from any destabilizing components present in the blood and to reduce toxicity, early studies investigated the encapsulation of $\mathrm{PQ}$ phosphate in the aqueous cavity of multilamellar vesicles (MLVs) [31]. A different biodistribution pattern was found as expressed by the higher concentrations in the target organ, the liver, and the more limited uptake and, consequently, toxicity in other organs. However, the therapeutic effect was not enhanced compared to the free drug, because the liposomes were taken up by the hepatic Kupffer cells and not by the hepatocytes [105].

At the beginning of the 1990s, the ability of QN or CQ to be encapsulated in neutral large unilamellar vesicles (LUVs) using also the $\mathrm{pH}$ gradient method was investigated [106]; uptake extents were 148 and $104 \mathrm{nmol} / \mu \mathrm{mol}$ lipid and 81 and $88 \mathrm{nmol} / \mu \mathrm{mol} \mathrm{lipid,} \mathrm{at}$ $15 \mathrm{~min}$ and $2 \mathrm{~h}$ time points, for QN and CQ, respectively. The sum of the results of the encapsulation of antimalarials in LUVs demonstrated that drugs such as QN and CQ are able to accumulate within these vesicles, which exhibit a proton gradient [107].

Artemether (AM), an antimalarial drug introduced in 1988 [108], has been encapsulated in neutral multilamellar liposomal formulations to be used by the oral route [109]. Evidence was found that the type of the phospholipid, as well as the incorporation of cholesterol in the liposomal bilayer, altered the AM entrapment efficiency, the size of the liposomes and the drug release rate. Multilamellar liposomes prepared with dibehenoylphosphatidylcholine (DBPC), CHOL and AM (1:1:2 molar ratio) presented a mean size of $3.20 \pm 1.03 \mu \mathrm{m}$ and a drug entrapment of 82.3\%. The AM release rate from liposomes prepared with mixtures of DBPC and dipalmitoylphosphatidylcholine (DPPC) (1:1 molar ratio) was $0.818 \%$ /day, while it was only $0.783 \%$ /day when CHOL was added to DBPC in a ratio of $1: 1$ (low $\mathrm{CHOL}$ ) and $0.616 \%$ when $\mathrm{CHOL}$ was used in a ratio of 1:2 (high $\mathrm{CHOL}$ ). These findings showed that the increase in the length of the acyl chain of the phospholipids as well as the addition of $\mathrm{CHOL}$ led to a decrease in the AM release rate. It is well known that $\mathrm{CHOL}$ molecules confer structural stability, providing a more ordered bilayer structure. The presence of CHOL may, therefore, induce drug/phospholipid interactions in the bilayer, which reduces drug release. Further studies were undertaken to evaluate the pharmacokinetic parameters of AM-loaded liposomal formulations following oral and intravenous administration in rabbits compared with an oral aqueous suspension [110]. Liposomes presented a $67.56 \%$ entrapment efficiency and particle size of $3.2 \pm 0.76 \mu \mathrm{m}$. The increase in AM bioavailability was evident in the case of orally administered liposomes with a relative bioavailability of $97.91 \%$, compared to $31.83 \%$ for the oral suspension. By the intravenous route, the same formulation promoted an increase in the half-life of the drug.
Recently AM has been encapsulated in neutral liposomes and its therapeutic efficacy evaluated in mice infected with $P$. chabaudi. An encapsulation efficiency of about $100 \%$ was achieved and formulations maintained their stability for 3 months at $4{ }^{\circ} \mathrm{C}$. A $100 \%$ cure of $P$. chabaudi-infected mice was observed after 22 days of infection [68].

Artesunate (AS), an artemisinin derivative, has also been encapsulated in neutral liposomes using pH 5 buffer solution as aqueous phase to prevent the aqueous instability of AS [111]. The AS-loaded liposomes presented an encapsulation efficiency of approximately $100 \%$ and remained stable for 10 days at $25^{\circ} \mathrm{C}$. The release of AS from the liposomes was influenced by the lipid content. In fact, the release rate decreased with the increase in lipid concentration.

More recently, the encapsulation of CQ in long-circulating liposomes was proposed and the drug release kinetics evaluated mimicking physiological fluids and endosome environments [52]. CQ was highly encapsulated in neutral conventional and PEGylated liposomes using a transmembrane $\mathrm{pH}$ gradient method to the improve encapsulation efficiency. Initially, blank conventional liposomes were prepared with SPC and CHOL, or PEGylated liposomes using 5 mol\% PEG 2000 grafted onto DSPE-PEG 2000 and CHOL. Next, the entrapment of the drug was performed in neutral preformed liposomes by an imposed $\mathrm{pH}$ gradient (acidic internal phase of liposomes). Finally, the CQ-loaded liposomes were lyophilized using trehalose as cryoprotectant. The highest CQ efficiency of encapsulation was $99 \%$ before lyophilization and $87 \%$ after hydration of the lyophilized form for an internal pH of 3.6 (0.2 M). The in vitro release profile of $\mathrm{CQ}$ was different, considering the $\mathrm{pH}$ of the internal phase of liposomes and the $\mathrm{pH}$ of the release medium (7.4 and 5.5). For conventional liposomes, the cumulative release of $\mathrm{CQ}$ at physiological $\mathrm{pH} 7.4$ was $30 \%$ within $6 \mathrm{~h}$, while more than $90 \%$ of the drug was released from liposomes at $\mathrm{pH}$ 5.5. In contrast, PEGylated liposomes significantly reduced the release of CQ from liposomes at pH 5.5.

\subsubsection{Conventional and long-circulating negatively-charged liposomes}

As QN and derivatives CQ and PQ are positively charged at physiological $\mathrm{pH}$, their encapsulation efficiency should be improved in negatively-charged liposomes. As a result, different conventional and long-circulating liposomal formulations were proposed as carriers for these antimalarials.

PQ was encapsulated in negatively-charged MLV with a CHOL-rich bilayers and the biodistribution of these systems was evaluated [112]. The encapsulation of PQ was around 13.4\% in liposomes consisting of PC, phosphatidylserine (PS) and CHOL (4:1:5 molar ratio) [113]. Plasma clearance, urinary excretion and tissue distribution of radiolabelled free PQ and PQ-loaded liposomes in mice were monitored for $2 \mathrm{~h}$ following intravenous administration. The results showed that $\mathrm{PQ}$-loaded liposomes were eliminated very rapidly from the plasma and excreted predominantly in the urine. A remarkable accumulation of the labelled drug occurred in liver $>$ kidneys $>$ lungs $>$ skeletal muscles. Partial erythrocytic sequestration results in higher and more persistent drug levels in blood cells than in plasma. Compared to the free drug form, PQ entrapped within CHOL-rich multilamellar liposomes exhibited a prolonged plasmatic half-life and excretion was reduced 8-fold, during the observation period. Liver accumulation of the labelled drug doubled, accounting for almost $50 \%$ of the injected dose; splenic uptake was increased 3-fold, while accumulation in the lungs, kidneys, heart and brain was drastically reduced. These differences in the pharmacodynamic behaviour may explain why liposomal entrapment leads to diminished acute PQ toxicity. Negatively-charged CQ-containing liposomes were prepared using DSPC and dipalmitoylphosphatidylglycerol (DPPG), leading to fluidor gel-state liposomes [89]. These liposomes were tested in P. bergheiinfected mice and compared to the performance of the free drug administered by the intraperitoneal (i.p.) route. An increase in therapeutic and prophylactic potential was found when CQ was encapsulated in fluid-state liposomes. Later, a further study was 
undertaken using the same model to demonstrate that the encapsulation of CQ into gel-state liposomes considerably enhanced the therapeutic effect [114]. CQ determinations in whole blood, plasma, and RBCs after i.p. administration of fluid- or gel-state CQ-loaded liposomes revealed a prolonged availability of the drug as compared to free $C Q$.

The influence of lipids, surface charge and the presence of CHOL on the biodistribution profile of PQ encapsulated in gel-state and liquidcrystalline state MLV has been investigated [113,115]. Several PQliposomal formulations were prepared with DPPC, cholesteylhemesuccinate (CHEMS), a charge inducer, and with or without CHOL in molar ratios of 7:1:(2) and 10:1:(4). Gel- (DPPC:CHEMS:CHOL and PC-100H:CHEMS:CHOL) and liquid-crystalline state $(\mathrm{PC}-100 \mathrm{H}$ : CHEMS:CHOL and PC-90G:CHEMS:CHOL) liposomes were prepared. The PC-100H:CHEMS:CHOL liposomes accumulated to a greater extent in the liver $(85.8 \%)$ than the other liposomes. The liquidcrystalline state PQ liposomes accumulated in the liver to a lower extent than the gel-state liposomes. From these results, it was concluded that the use of PQ-loaded liposomes, prepared with PC $100 \mathrm{H}, \mathrm{CHEMS}$ and $\mathrm{CHOL}$, protects non-target tissues such as lung, kidneys, heart and brain from PQ accumulation, thereby reducing the toxicity of $\mathrm{PQ}$.

The efficacy of liposomal desferroxamine B (DFO), an iron chelator, has been studied in P. vinckei-infected mice [116]. Negatively-charged liposomes containing DFO were obtained using EPC and PG or DSPC and DSPG in the presence or absence of CHOL. P. vinckei-infected mice were exposed to three different administration regimens: (i) multiple subcutaneous injections of free DFO, (ii) i.p. infusion of free DFO, or (iii) multiple subcutaneous injections of liposomal DFO. First, the effect of prior treatment with free and liposomal DFO (administered 1 day before infection) on the immune system of animals was evaluated. Then, multiple subcutaneous injections of free DFO before and after infection suppressed parasitemia, whereas injections only prior to infection did not. Suppression of parasitemia and long-term survival (1 month after infection) of mice were obtained by i.p. infusion starting 1 day before infection (14 days, $130 \mathrm{mg} \mathrm{DFO} / \mathrm{kg} /$ day) or by subcutaneous injections of liposomal DFO prior to infection (days 21 and 0,400 or $800 \mathrm{mg}$ DFO $/ \mathrm{kg} /$ day). The efficacy of the antimalarial activity of liposomal DFO was influenced by the drug-to-lipid ratio but was hardly affected by the bilayer rigidity. Finally, DFO when treatment was started 6 and 7 days after infection, parasitemia was reduced by all three treatment regimens. The results revealed that liposomes are suitable for carrying DFO, which should be given prior to infection and continued during the advanced stages of the experimental malaria. Unexpectedly, parasitemia was increased after subcutaneous (s.c.) injections of free DFO prior to infection. Based on previous studies, it was suggested that at low doses of DFO iron chelation impairs part of the immune system, whereas the effect on parasiteassociated iron is negligible, resulting in an increase in parasitemia. On the other hand, at high doses or by multiple administration of DFO, the effect of chelation on parasite-associated iron by DFO is predominant, resulting in the suppression of parasitemia.

Recently, to circumvent the stability problems of liposomes, an innovation in PQ-liposomal dosage forms has been proposed. The drug encapsulation occurred during the hydration of the lyophilized form of conventional and long-circulating liposomes [117]. As PQ is an amphoteric drug (pKa 3.2 and 10.4) with pH-dependent solubility, it was successfully encapsulated in conventional negatively-charged liposomes. Once obtained, liposomes were lyophilized and sterilization by $\gamma$-irradiation was performed. The $\mathrm{pH}$ gradient was maintained during the lyophilization-rehydration process. DSPC-based liposome preparation with high $\mathrm{CHOL}$ content and citrate concentration and low drug concentration showed high encapsulation efficiency (96\%). The final preparation consisted of a three-vial kit with solid-state PQ lyophilized liposomes and hydration medium, where the PQ encap- sulation was assumed to take place during the hydration, immediately before the use of liposomes. Unfortunately, no efficacy studies of this formulation have been reported to date.

The concept of polysaccharide-coated liposomes has been used to encapsulate QN in negatively-charged surface liposomes consisting of PC and PG [118]. Chitosan-coated liposomes, known as chitosomes, were obtained by putting the liposomes in contact with a chitosan solution at a suitable $\mathrm{pH}$. The $\mathrm{pH}$ gradient method was able to increase the encapsulation of $\mathrm{QN}$ in liposomes. QN-loaded chitosomes presented a size of $200-250 \mathrm{~nm}$ and maintained their stability when stored at $4{ }^{\circ} \mathrm{C}$ for at least 30 weeks. The released amount of quinine from PC/PG liposomes were $34.3 \pm 1.7 \%$ at the first measurement and $89.4 \pm 6.0 \%$ after 6 days ( $C V=0.6-9.0 \%$ ). The release of $\mathrm{QN}$ from chitosomes was quite similar: $41.7 \pm 0.6 \%$ at the first measurement and $97.7 \pm 2.6 \%$ at the end of the experiment $(n=3, \mathrm{CV}=0.6-2.6 \%)$. The difference in the initial burst effect for the two formulations was explained by the release of the adsorbed QN molecules when the liposomes are diluted. It is also possible that chitosan displaces QN molecules on the membrane surface, thereby producing a high initial release.

\subsubsection{Targeted liposomes for antimalarials}

Based on the concept of pro-drugs, PQ has been linked to leucine and to peptides (alanyl-leucine and alanyl-leucyl-alanyl-leucyl) as intermediate steps in the synthesis of covalent PQ-glycoprotein conjugates to be selectively recognized by hepatocytes [87]. These pro-drug forms of $\mathrm{PQ}$ were entrapped in liposomes and the therapeutic activity was tested in mice infected with $P$. berghei sporozoites. Causal prophylactic cures were obtained after a single intravenous injection of PQ-liposomes $(60-70 \mathrm{mg} \mathrm{PQ} / \mathrm{kg}$ of bodyweight) and lower doses (35 mg PQ/kg of bodyweight) of ala-leu-PQ and ala-leu-ala-leu-PQ. The improved therapeutic index of ala-leuPQ and ala-leu-ala-leu-PQ derivatives was a result of their decreased toxicity and increased chemotherapeutic activity. The administration of such high doses was only possible because of the decreased toxicity of PQ entrapped in liposomes and confirms the validity of nanocarrier systems for the treatment of malarial infections.

5.1.3.1. Peptide-targeted liposomes. Recently, the targeting of liposomes to hepatocytes using the peptide surface-bearing approach has been studied more thoroughly. An investigation of peptide-targeted and long-circulating liposomes for further application in malaria treatment was conducted using a 19-amino-acid sequence from the $\mathrm{N}$-terminal region of a protein expressed by $P$. berghei circumsporozoite [69]. The peptide was chemically bound to the surface of PEGylated liposomes prepared with labelled lipids. Bioavailability studies demonstrated that liposomes were one 100 -fold more targeted to hepatocytes and non-parenchyma liver cells than to the heart, lungs and kidneys, and 10-fold more than to the spleen. These promising findings indicated that it is feasible to target antimalarial drugs to combat parasites in hepatocytes using peptide-targeted liposomes. Later, following the development of different formulations of PEGylated and PEGylated-peptide liposomes, their in vivo evaluation of was carried out [85]. The in vivo ability of liposomal formulations to target the liver was confirmed, with approximately $80 \%$ of the total injected dose recovered in the liver within $15 \mathrm{~min}$. The uptake of peptide-bearing PEGylated liposomes by liver cells was more than 600-fold higher than the uptake in the heart, and more than 200-fold higher than the uptake by lung or kidney cells. Furthermore, the successful effective targeting of liposomes to the liver was achieved with a multiple-dose regimen (up to three administrations) over 42 days at 14-day intervals. An interesting finding was the fact that the most cost-effective formulation was obtained with the minimal use of peptide and polymer compounds. These PEGylated-peptide coated liposomes present a great potential for targeting antimalarial drugs to the liver. 
5.1.3.2. Antibody-bearing liposomes. The binding of antibodies to the surface of liposomes to target antimalarials to erythrocytes has been the subject of several studies [86-88,90]. CQ was encapulated into MAb F $\mathrm{F}_{10^{-}}$ bearing liposomes and its efficacy was evaluated in mice infected with CQ-susceptible or CQ-resistant P. berghei [70]. CQ-loaded MAb F $10^{-}$ liposomes were able to control not only the CQ-susceptible but also the CQ-resistant $P$. berghei infections. The treatment with CQ-loaded MAb $\mathrm{F}_{10}$-liposomes by intravenous injection at a dose of $5 \mathrm{mg} / \mathrm{kg}$ of body weight per day on days 4 and 6 post-infection completely cured the mice infected with CQ-resistant $P$. berghei (75\% and 90\%, respectively). The high therapeutic efficacy of liposomal CQ was attributed to the high degree of specific interaction of the MAb $\mathrm{F}_{10}$-fragment with the Plasmodium-infected erythrocytes, the efficient internalization of the liposomes by infected cells and the specific binding of $\mathrm{MAb} \mathrm{F}_{10^{-}}$ liposomes to parasites. The uptake of $\mathrm{MAb} \mathrm{F}_{10}$-liposomes provided a high concentration of the drug in infected cells not only by the selective recognition of these cells but also by the ability of liposomes to deliver several drug molecules at the same time to the target cells.

Antibody-bearing liposomes were prepared by covalent attachment of anti-erythrocyte $\mathrm{F}\left(\mathrm{ab}^{\prime}\right)_{2}$ to the surface of liposomes. Antibody-targeted liposomes induced a considerable enhancement in the liposome binding to erythrocytes in vivo and have been proved to be effective vehicles for delivering the CQ to erythrocytes in $P$. berghei-infected mice [90].

On the basis of the studies reported above, liposomes have been extensively used in antimalarial experimental therapy for the passive and active targeting to Plasmodium-infected erythrocytes or hepatocytes by acting as a local depot. They reduced toxicity, improved therapeutic efficacy against malaria parasites, modified the pharmacokinetics, and ensured prolonged in vivo release of drugs.

\subsection{Liposomes as adjuvants for malaria vaccines}

Several liposomal antimalarial vaccines using different strategies such as encapsulated antigens and synthetic peptide vaccines are currently under clinical investigation. For more details, the reading of a number of recent reviews on this subject is recommended $[26,119,120]$. Liposome-based vaccines for malaria have the further potential to induce both antibody and cellular immune response by simultaneously activating the major histocompatibility complex (MHC) class I and MHC class II pathways.

Liposomes containing lipid A have been extensively studied as potent adjuvants for malaria vaccines [26,101,121,122]. In fact, it was reported that liposomes containing lipid A induced potent humoral immune responses to the encapsulated malaria antigen (R32NS1) in mice [123]. Negatively-charged MLV-liposomes were composed of dimyristoylphosphatidylcholine (DMPC), PG, CHOL, and lipid A in molar ratios of 1.8:0.2:1.5:0.04 (Limulus positive) or 1.8:0.2:1.5:0.005 (Limulus negative). The immune response was not enhanced by lipid $A$ alone or by empty liposomes containing lipid A. Liposomes containing lipid A and R32NS1 also activated gamma interferon-treated macrophages to produce nitric oxide. Macrophage activation and antigen presentation occurred with Limulus negative liposomes. The i.p. injection of liposomal lipid A caused a marked increase in the recruitment of immature (peroxidase-positive) macrophages to the peritoneum. Based on these experiments, the mechanism of the adjuvant action of liposomal lipid A is partly due to an increased antigen expression by macrophages and partly to the recruitment of an increased number of macrophages serving as antigen-presenting cells.

The immunization of BALB/c mice with conventional liposomes prepared with EPC and CHOL (2:1 molar ratio) containing 66pyIMP, an integral protein from a multi-drug-resistant strain of $P$. yoelii nigeriensis, was able to protect animals against lethal $P$. yoelii infection [124]. The highest levels of IgG, IgG1 and IgG2a were produced in animals immunized with liposomal 66PyIMP as compared with the response of the animals treated with the emulsified form of 66PyIMP in Freund's adjuvant and that of the control animals receiving phosphate saline solution. Furthermore, the immunization with 66PyIMP-loaded liposomes eliminated parasites from the systemic circulation within 6 days ( $0.28 \%$ parasitemia count).

\subsection{Solid lipid nanoparticles as nanocarriers for antimalarials}

Transferrin (Tf)-conjugated solid lipid nanoparticles (SLNs) were investigated for their ability to target QN dihydrochloride to the brain for the management of cerebral malaria [71]. QN-loaded Tf-SLNs were developed by an ethanol injection method using hydrogenated soya phosphatidylcholine (HSPC), triolein, CHOL and DSPE. In vitro fluorescence studies revealed an enhanced uptake of Tf-SLNs in brain tissue compared with non-conjugated SLNs. In vivo studies evaluated the QN plasma level and tissue distribution after intravenous administration of QN-loaded Tf-SLNs and unmodified SLNs compared to that of free drug. The intravenous administration of QN dihydrochloride solution resulted in higher drug concentrations in the serum than with SLNs. Also, Tf conjugation significantly enhanced the brain uptake of $\mathrm{QN}$, which was shown by the recovery of a higher percentage of the dose from the brain following administration of Tfcoupled SLNs compared with conventional SLNs or drug solution.

The low oral bioavailability of AM, about $40 \%$ (Table 1 ), is due to its poor aqueous solubility and degradation in the acidic environment of the stomach. On the other hand, the oily intramuscular injection of $\mathrm{AM}$ is not very suitable when rapid eradication of the severe malarial infections is required. As a result, nanostructured lipid carriers (NLC) containing AM were reported recently [125]. The NLC are lipid nanocarriers based on the mixture of biocompatible solid lipid and liquid lipid (oil) [126]. AM-loaded NLC, known as Nanoject ( $60 \mathrm{~nm}$ ), was formulated by employing a microemulsion template technique [125]. The antimalarial activity of the AM-Nanoject and a conventional AM injectable formulation was evaluated in P. berghei-infected mice. AM was released from Nanoject in a sustained manner. AM-Nanoject showed significant antimalarial activity as compared to the marketed injectable formulation (Larither $\left.{ }^{\circledR}\right)$. AM-Nanoject produces an improved animal survival rate in comparison with the oily formulation. However, the formulations were compared by the intraperitoneal route, not by intravenous one, and it is generally agreed that small NLC extravasate faster to the blood, thus improving AM pharmacological effect.

\subsection{Nano and microemulsions as carriers for antimalarials}

The nanoemulsions ( $\mathrm{NE})$, oil-in-water $(\mathrm{O} / \mathrm{W})$ emulsions with a mean diameter of $\leq 500 \mathrm{~nm}$ (on the nanometric scale), were also investigated as antimalarial carriers. NE presented two important advantages over the other colloidal carriers, namely their low cost and availability as oral dosage forms. Considering these advantages, a reconstituted artificial chylomicron NE containing PQ was proposed for targeting hepatocytes following intravenous injection [127]. The PQ was encapsulated into NE in a highly efficient manner and remained inside them without any significant release. Moreover, when intravenously injected into mice, the PQ-loaded chylomicron NE led to a significantly enhanced accumulation of $\mathrm{PQ}$ in the liver, when compared to the injection of free $P Q$.

Recently, an O/W NE prepared with Miglyol ${ }^{\circledR}$ and used to encapsulate PQ for oral administration has been reported [128]. NE improved the antimalarial activity of PQ at a lower dose than that of the PQ solution form in P. berghei-infected mice. A reduction of $25 \%$ in the dose level of PQ was achieved using the NE dosage form $(1.5 \mathrm{mg} /$ $\mathrm{Kg} / \mathrm{day}$ ), which produced a satisfactory suppression of parasitemia (92.75\%) and a mean survival time of 34.9 days. Moreover, NE achieved significantly higher PQ levels in the liver after oral administration, corroborating the enhanced antimalarial activity of $\mathrm{PQ}$ by improving drug concentration at the site of action. 
More recently, a practical approach to treating experimental malaria was proposed in the form of self-microemulsifying drug delivery systems (SMEDDS), which, after administration by the oral route, are rapidly transformed into microemulsions [129]. SMEDDS can generally be used to fill conventional hard gelatin capsules and may improve the bioavailability of poorly water-soluble drugs. SMEDDS were developed with long chain triglycerides (N-LCT) as an oily phase containing AM. This new formulation (AM-SMEDDS) resulted in a significant improvement in the antimalarial activity of AM against $P$. berghei-infected mice, with an average parasitemia of $35.11 \pm 4.16$ on day 20 of infection as compared to that of Larither ${ }^{\circledR}$, a marketed capsule dosage form containing AM, with an average parasitemia of $42.35 \pm 4.18(p<0.05)$.

Moreover, a solid microemulsion pre-concentrate formulation, referred to as NanOsorb, has been recently reported as a safe and efficacious oral delivery strategy for the use of AM in the treatment of experimental malaria [130]. The AM-NanOsorb system significantly improved the therapeutic efficacy of AM in P. berghei-infected mice as compared to the treatment with AM solution and the marketed formulation Larither®. The antimalarial activity of AM-NanOsorb was 2.6-fold and 2.3-fold higher than that of AM solution and Larither ${ }^{\circledR}$, respectively. Results of AM-SMEDDS and AM-NanOsorb are probably explained by the enhanced AM solubilization in the gastrointestinal tract induced by the oily vehicles, thus improving drug bioavailability.

\section{Polymeric-based nanocarriers for antimalarials}

\subsection{Polymeric nanoparticles as nanocarriers for antimalarials}

Polymeric nanospheres (NS) constitute the most thoroughly investigated nanocarriers for drug delivery, after liposomes [20,63,131]. The surface properties of the NS can be modulated using a wide variety of polymers in order to obtain passive or active targeting in the body (Fig. 4). Due to their polymeric nature, NS generally possess high stability in biological fluids and under stress conditions of preparation and storage, which qualifies them as promising drug delivery systems for antimalarials. However, when compared with other drugs, fewer reports have been published on the delivery of antimalarial drugs using polymeric systems such as NS.

The only report found on antimalarial drugs discusses the entrapment of PQ in albumin-NS prepared by a combined emulsification and congealing procedure [132]. However, these formulations were not tested in vivo. One initial attempt has been made to demonstrate the susceptibility of $P$. falciparum to antisense oligodinucleotide nanoparticles (ODN-NS) [72]. This interesting approach used antisense ODNchitosan NS about $50 \mathrm{~nm}$ in size to improve antisense ODN internalization by $P$. falciparum-infected erythrocytes through erythrocyte permeation pathways (Fig. 3B). The target was the malarial topoisomerase II gene [72]. Both negatively and positively charged antisense ODNchitosan NS, as well as free antisense ODNs in a concentration of $0.5 \mu \mathrm{M}$, showed a sequence-specific inhibition compared with sense sequence controls. However, ODN-chitosan NS were much more sequencespecific in their antisense effect than free ODNs. ODN-chitosan NS with a negatively-charged surface exhibited a significantly stronger inhibitory effect (87\%) on the P. berghei growth in comparison with the positive ones (74\%) or free ODNs (68\%). To our knowledge, this is the first study demonstrating the susceptibility of $P$. falciparum to antisense ODN-NS.

\subsection{Dendrimers as nanocarriers for antimalarials}

Artemether, a potent and very hydrophobic antimalarial drug, was solubilized in dendrimers. A long-circulating and site-specific system was prepared with PEG-lysine type dendrimer and chondroitinsulfate A (CSA) for sustained and controlled delivery of the drug via the intravenous route of administration [133]. These systems entrapped 10-18 molecules of AM per molecule of dendrimer. The release was prolonged up to 1-2 days; depending upon the increase in polymer generation from 4.0 to $5.0 \mathrm{G}$. Conjugation with equimolar CSA increased the amount of drug loading and prolonged drug release significantly 2- to 3-fold. CSA coating made the dendrimer system relatively less hemeolytic and larger in particle size, and significantly decreased macrophage toxicity. In addition, CSA coating of the dendrimer system helped in clearing the circulating ring and trophozoite stages of $P$. falciparum in in vitro cultures. In vivo studies showed a prolonged release of AM from both CSA-coated and uncoated dendrimer systems up to $13 \mathrm{~h}$. The mean residence time was found to have increased nearly 2 - and 4 -fold, respectively, from those uncoated and coated-dendrimer systems, as compared to injected AM solutions. CSA-coated dendrimer systems seem to act as sustained-release circulating nanocarriers of antimalarials.

Subsequently, the use of uncoated and CSA-coated poly-l-lysinebased dendrimer systems for controlled and sustained delivery of CQ phosphate was also proposed [134]. In that study, there was also a significant reduction in in vitro levels of the ring and trophozoite stages of $P$. falciparum when treated with CSA-coated lysine-based dendrimers. In vivo studies in albino rats have indicated that these systems are suitable for prolonging and controlling the blood level of CQ after intravenous administration. It has been suggested that CSAcoating is able to improve drug-loading capacity and that it can control and sustain the release of CQ from such carriers. It is also suitable for use as a safe and effective carrier for intravenous CQ administration. Furthermore, the loading of PQ in long-circulating PEG-lysine type dendritic peptide-based macromolecular carrier has been analyzed for sustained and controlled delivery [135]. PEG-amine was used as the core in the proposed systems and the various generations of dendrimers were synthesized. It was observed that there was no stimulation of the macrophage level or white blood cell (WBC) count using the PEGylated PQ-dendrimers. In addition, RBC counts remain closer to normal values in the case of drug delivery by PEGylated systems as compared to long-term free PQ delivery [135]. However, despite these interesting results, no studies on the efficacy of dendrimer nanocarriers in experimental malaria have been carried out to date.

\section{Other antimalarial nanocarriers}

Numerous new drug candidates are excluded because they are not soluble in the initial in vitro screening tests against Plasmodium strains, since solubility is a limiting requirement for in vitro activity evaluation. However, some of these new chemical entities are active when formulated in nanostructured dosage forms, such as nanosuspensions, micelles, cyclodextrin inclusion complexes or even lipid and polymeric carriers. In view of this, a number of attempts have been made to use cyclodextrins and nanosuspensions as solubilizing agents and carriers for antimalarial drugs.

\subsection{Cyclodextrins and inclusion complexes with antimalarials}

The use of cyclodextrines (CDs), cyclic oligosaccharides, is the most widely employed approach to improving drug solubility and bioavailability as drug:inclusion complexes [136]. Low cost and biocompatibility of cyclodextrins may be the main reasons for their use as a drug solubilizer. Earlier studies of complexation of antimalarials with CDs showed that the oral bioavailability of artemisinin was improved by $\beta$ - or $\gamma$-CD inclusion complexes [137]. Findings indicated that the artemisinin: $\beta-C D$ or artemisinin: $\gamma-C D$ complexes had a much greater bioavailability than Artemisinin 250®. In addition, it has been suggested that sulphated-CDs inhibit Plasmodium growth by interacting with the anion transport protein, AE1 [138]. Furthermore, sulphated-CDs were found to be active against $P$. falciparum cultures and to inhibit $P$. berghei merozoite entry in RBC. 
Many other studies have reported the complexation of antimalarials with CDs with a view to improving their solubility [139-141]; however, only the physicochemical aspects of the drug-inclusion complexes were studied.

\subsection{Nanosuspensions as carriers for antimalarials}

Nanosuspensions are sub-micron colloidal dispersions of a pure poorly water-soluble drug stabilized by surfactants, without any matrix material suspended in dispersion [142,143]. Only one report of an antimalarial drug encapsulated in nanosuspension was found [144]. A nanosuspension of dihydroartemisinin (DHA) was successfully formed after dispersing ternary ground mixtures of polyvynilpyrolidone (PVP), sodium deoxycholate (NaDC) and DHA (PVP/ $\mathrm{DHA} / \mathrm{NaDC}$ ) or $\mathrm{DHA} / \mathrm{NaDC}$ ground mixtures in water using a vibrating rod mill. The ternary ground mixtures did not yield an improved nanosuspension in terms of particle size reduction or recovery of drug particles, but they provided more physically stable nanosuspensions than DHA/NaDC ground mixtures. The size of the DHA-nanosuspensions decreased with the increase in grinding time and in the presence of a small amount of PVP and NaDC. About $95 \%$ of the drug particles were found in the DHA-nanosuspensions. Zeta potential measurement suggested that the stability of DHA-nanosuspensions was due to the adsorption of NaDC and PVP onto the surface of the drug particles. Atomic force microscopy and transmission electron microscopy indicated that the drug was in a nanocrystal form. In addition, DHA-nanosuspensions presented higher in vitro antimalarial activity against $P$. falciparum than microsuspensions. These results suggest that the co-grinding of DHA with PVP and NaDC seems to be a promising method of preparing DHA-nanosuspensions.

\section{Nanocapsules: promising polymeric-lipid nanocarriers}

Nanocapsules are defined as complex carriers composed of a polymeric wall surrounding an oil core, into which lipophilic drugs can be encapsulated [145]. The high entrapment efficiencies of lipophilic drugs, their low polymer content and low inherent toxicity are the main advantages of NC compared to other nanocarriers $[19,146]$. The therapeutic advantages of NC include solubilization of poorly watersoluble drugs, protection against drug inactivation in the gastrointestinal tract, protection against drug toxicity $[33,147,148]$, enhanced permeation of drugs through mucosal surfaces and prolongation of the blood circulation of injected drugs [83]. Since the mid-1980s, different active molecules have been encapsulated in NC [148]. However, to our knowledge, there are few reported investigations addressing the potential of nanocapsules in the delivery of antimalarial drugs.

Our research team has been working with conventional and longcirculating $\mathrm{NC}$ to encapsulate Hf a very lipophilic antimalarial drug $[32,33,85]$. Our main goal is the development of a stable injectable NC formulation containing $\mathrm{Hf}$ to be tested in an experimental model of malaria. Hf-NC was prepared from biodegradable polymers and medium chain triglycerides as the oily core. PLA and its copolymer conjugated with PEG (PLA-PEG) were used to prepare Hf-NC with different surface properties. Highly efficient loading of the $\mathrm{Hf}$ free base form was obtained in PLA-NC 150-250 nm in size. Hf-NC with high density PEG chains $(20 \mathrm{kDa})$ covalently grafted at their surface were the most stable formulations [84]. Hf release from NC occurred mainly by partition with the external medium. The addition of serum to the release medium allowed complete and rapid Hf release from PLA-NC because of the high affinity of $\mathrm{Hf}$ for serum proteins. However, the presence of covalently-grafted PEG chains at the surface of PLA-NC limited Hf release by providing a hydrophilic steric barrier at the particle surface. A dense coverage of PLA-NC with PEG long chains provided the best control of Hf release. The efficacy and pharmacokinetics of this new Hf-NC parenteral formulation were evaluated in mice infected with $P$. berghei [32]. No toxic effects were observed for Hf-NC after intravenous administration for doses of up to $100 \mathrm{mg} / \mathrm{kg}$, whereas the solubilized form of Hf in PEG-dimethylacetamide was very toxic at this dose. Furthermore, Hf-NC showed antimalarial activity similar to or better than that of the Hf solution in the 4-day test at a single dose in severely infected mice, with only minimal differences between the conventional and long-circulating NC formulations. Hf pharmacokinetics was evaluated during parasite development in severely $P$. berghei-infected mice. The nanoencapsulation of Hf increased the AUC more than 6-fold, compared with the $\mathrm{Hf}$ solution, throughout the experimental period of $70 \mathrm{~h}$. In addition, Hf$\mathrm{NC}$ induced a significantly faster control of parasite development than the $\mathrm{Hf}$ solution in the first $48 \mathrm{~h}$ post-treatment. While the parasitemia fell more rapidly with PLA-NC, the effect was more sustained with the surface-modified PEG-PLA-NC. This was consistent with the fact that PEG-PLA-NC remained longer in the circulation. These results showed that Hf-NC formulations may provide a more favourable Hf plasma profile, reduce the intravenous dose and thus reduce drug toxicity [32]. The use of Hf-NC by the parenteral route in the treatment of severe malaria is, therefore an approach worth exploiting.

Recently, the toxicity of the Hf-NC prepared with poly- $\varepsilon$ caprolactone $(\mathrm{PCL})$ has been reported in detail, because Hf chloridrate ( $\mathrm{Hf} . \mathrm{HCl})$ is frequently associated with QT interval prolongation in the electrocardiogram. The acute lethal dose $\left(\mathrm{LD}_{100}\right)$ of $\mathrm{Hf} . \mathrm{HCl}$ experimentally observed was $200 \mathrm{mg} / \mathrm{kg}$, whereas the calculated $\mathrm{LD}_{50}$ was $154 \mathrm{mg} / \mathrm{kg}$. In contrast, the $\mathrm{LD}_{100}$ for Hf-NC was $300 \mathrm{mg} / \mathrm{kg}$ with a longer mean time to death than Hf.HCl. The Hf entrapped in PCL NC presented a greater efficacy than both PLA-PEG NC and Hf solution in $P$. berghei-infected mice at $1 \mathrm{mg} / \mathrm{kg}$. The cardiovascular parameters, ECG and arterial blood pressure were evaluated in anaesthetized Wistar rats after the IV administration of a single, especially high dose (100 and $150 \mathrm{mg} / \mathrm{kg}$ ) of Hf-NC or Hf.HCl solution. It was observed that Hf solution caused prolongation of the QT and PR intervals of the ECG; however, this effect was significantly $(p<0.001)$ reduced when Hf was administered entrapped in NC. The treatment with $\mathrm{Hf} . \mathrm{HCl}$ induced a pronounced bradycardia and severe hypotension leading to death. The effect of Hf-NC upon heart rate was reduced from $58 \%$ to $75 \%$ for 100 and $150 \mathrm{mg} / \mathrm{kg}$, respectively, when compared with $\mathrm{Hf} . \mathrm{HCl}$ solution. These findings showed that the encapsulation of $\mathrm{Hf}$ reduced the QT interval prolongation of ECG in rats and suggested that a modification of drug distribution is feasible using NC. Hf encapsulation was the main factor responsible for the significant reduction in cardiac toxicity [33]. To date, this sequence of papers on $\mathrm{Hf}$ encapsulation is the most comprehensive investigation of antimalarial nanotechnological formulations for intravenous administration.

More recently, QN in its basic form was encapsulated in NC [73]. QN-NC with $200 \mathrm{~nm}$ in size were evaluated in vivo in a newlydeveloped $P$. berghei-Wistar rat experimental model. This model allows antimalarial pharmacokinetic-pharmacodynamic evaluation in Plasmodium-infected rats [149]. QN-NC efficacy was evaluated using different dosing regimens, and pharmacokinetics was evaluated after the intravenous administration of $25 \mathrm{mg} / \mathrm{kg}$ to infected rats. Survival improved with reduced doses of QN-NC. The mechanism responsible for the increased efficacy of QN-NC was the increased interaction between QN and erythrocytes, as suggested [73]. However, the pharmacokinetic parameters of QN-NC were not significantly different from those determined for free $\mathrm{QN}(p=0.05)$.

Furthermore, triclosan, a non-ionic broad-spectrum antimicrobial drug, which presents in vitro effectiveness in the inhibition of the growth of $P$. falciparum, has been encapsulated in chitosan-coated NC [150]. Chitosan-coated NC was obtained with chitosan hydrochloride at two different degrees of viscosity ( $\mathrm{Cl} 113$ and $\mathrm{Cl} 213)$. $\mathrm{Cl}$ 113-coated NC appeared to be particularly suitable as triclosan carriers for obtaining a systemic drug release, owing to the good mucoadhesive properties of chitosan, as well as the effectiveness shown by the coating in adequately controlling the drug release rate. 


\section{Conclusions}

Pharmaceutical nanotechnology could provide unlimited opportunities for improving the efficacy of the currently used antimalarial drugs and the new chemical entities characterized by poor solubility, chemical instability, inadequate bioavailability profile and toxicity. The wide range of modulations of the surface properties of these nanometric carriers aimed at improving antimalarial selectivity in the recently-discovered parasite targets has been little exploited to date. From this review, it emerged that nanotechnology applied to malaria therapy is a domain that is still in its infancy. However, a deep analysis of aspects involving the biology of the parasite, epidemiology of malaria, cost-effectiveness and social impacts of the new therapies is required. In order to successfully travel this long road it is essential to assemble highly interdisciplinary researchers with expertise in the areas of parasitology, intracellular trafficking, biomaterials, polymer science, nanotechnology, pathology, toxicology, and molecular and cellular immunology.

It is clear from this review that there is much to be exploited in the domain of polymeric nanoparticles in malaria therapy. Although a great deal of research has been focused on investigating liposomes, relatively less research has been undertaken with polymeric systems, particularly with surface modification for improving antimalarial drug targeting. It is expected that this review will eventually lead to a better understanding of malaria disease and provide insights into new strategies for developing smart, well-tolerated, low-cost and efficacious therapeutic targeted nanosystems to treat malaria-infected patients.

\section{Acknowledgements}

NSSM is grateful to the Brazilian National Council for Scientific and Technological Development $(\mathrm{CNPq})$ and the Brazilian Nanobiotechnology Network-Nanobiotec/MCT/CNPq for financial support. VCFM is grateful for the financial support received from WHO/TDR grant A00790, Nanobiotechnology Network-FAPEMIG and the Brazilian Nanobiotechnology Network-Nanobiotec/MCT/CNPq. The contributions of J.M. Rabanel and V. Aoun are also acknowledged in revising this manuscript.

\section{References}

[1] WHO, World Malaria Report (2008), http://www.who.int/malaria/wmr2008/ malaria2008.pdf, accessed on 15th April 2009.

[2] F. Gardella, S. Assi, F. Simon, H. Bogreau, T. Eggelte, F. Ba, V. Foumane, M.C. Henry, P.T. Kientega, L. Basco, J.F. Trape, R. Lalou, M. Martelloni, M. Desbordes, M. Baragatti, S. Briolant, L. Almeras, B. Pradines, T. Fusai, C. Rogier, Antimalarial drug use in general populations of tropical Africa, Malaria J. 7 (2008).

[3] WHO, The Africa Malaria Report (2003), http://www.rollbackmalaria.org/ amd2003/amr2003/pdf/amr2003.pdf, accessed on 15th June 2009.

[4] D. Rathore, T.F. McCutchan, M. Sullivan, S. Kumar, Antimalarial drugs: current status and new developments, Expert Opin. Invest. Drugs 14 (2005) 871-883.

[5] L.A. Smith, C. Jones, S. Meek, J. Webster, Review: provider practice and user behavior interventions to improve prompt and effective treatment of malaria: do we know what works? Am. J. Trop. Med. Hyg. 80 (2009) 326-335.

[6] WHO, Global Malaria Control and Elimination (2008), http://apps.who.int/ malaria/docs/elimination/MalariaControlEliminationMeeting.pdf, accessed on 30th June 2009

[7] P. Winstanley, S. Ward, Malaria chemotherapy, Adv. Parasitol. 61 (2006) 47-76

[8] A.U. Krettli, V.F. Andrade-Neto, M.G.L. Brandão, W.M.S. Ferrari, The search for new antimalarial drugs from plants used to treat fever and malaria or plants randomly selected: A review, Mem. I. Oswaldo Cruz 96 (2001) 1033-1042.

[9] B.M. Greenwood, D.A. Fidock, D.E. Kyle, S.H.I. Kappe, P.L. Alonso, F.H. Collins, P.E Duffy, Malaria: Progress, perils, and prospects for eradication, J. Clin. Invest. 118 (2008) 1266-1276

[10] A.U. Krettli, J.O. Adebayo, L.G. Krettli, Testing of natural products and synthetic molecules aiming at new antimalarials, Curr. Drug Targets 10 (2009) 261-270.

[11] T.S. Skinner-Adams, J.S. McCarthy, D.L. Gardiner, K.T. Andrews, HIV and malaria co-infection: interactions and consequences of chemotherapy, Trends Parasitol. 24 (2008) 264-271.

[12] J. Whitworth, D. Morgan, M. Quigley, A. Smith, B. Mayanja, H. Eotu, N. Omoding, M. Okongo, S. Malamba, A. Ojwiya, Effect of HIV-1 and increasing immunosuppression on malaria parasitaemia and clinical episodes in adults in rural Uganda: A cohort study, Lancet 356 (2000) 1051-1056.

[13] A.E. Greenberg, W. Nsa, R.W. Ryder, M. Medi, M. Nzeza, N. Kitadi, M. Baangi, N. Malanda, F. Davachi, S.E. Hassig, Plasmodium falciparum malaria and perinatally acquired human immunodeficiency virus type 1 infection in Kinshasa, Zaire - a prospective, longitudinal cohort study of 587 children, New Engl. J. Med. 325 (1991) 105-109.

[14] K. Grimwade, N. French, D.D. Mbatha, D.D. Zungu, M. Dedicoat, C.F. Gilks, Childhood malaria in a region of unstable transmission and high human immunodeficiency virus prevalence, Pediatr. Infect. Dis. J. 22 (2003) 1057-1063.

[15] J.G. Breman, C.V. Plowe, A malaria vaccine for control: more progress, J. Infect. Dis. 200 (2009) 317-320.

[16] K.E. Kester, J.F. Cummings, O.A. O., C.F. Ockenhouse, U. Krzych, P. Moris, R. Schwenk, R.A. Nielsen, Z. Debebe, E. Pinelis, L. Juompan, J. Williams, M. Dowler, V.A. Stewart, R.A. Wirtz, M.C. Dubois, M. Lievens, J. Cohen, W.R. Ballou, D.G.J. Heppner, S.V.E.G. RTS, randomized, double-blind, phase 2a trial of falciparum malaria vaccines RTS,S/ AS01B and RTS,S/AS02A in malaria-naive adults: safety, efficacy, and immunologic associates of protection, J. Infect. Dis. 200 (2009) 337-346.

[17] P. Mettens, P.M. Dubois, M.A. Demoitié, B. Bayat, M.N. Donner, P. Bourguignon, V.A. Stewart, D.G. Heppner Jr., N. Garçon, J. Cohen, Improved T cell responses to Plasmodium falciparum circumsporozoite protein in mice and monkeys induced by a novel formulation of RTS, S vaccine antigen, Vaccine 26 (2008) 1072-1082.

[18] D.J. Bacon, R. Jambou, T. Fandeur, J. Le Bras, C. Wongsrichanalai, M.M. Fukuda, P. Ringwald, C.H. Sibley, D.E. Kyle, World antimalarial resistance network (WARN) II: In vitro antimalarial drug susceptibility, Malaria J. 6 (2007) 1-8.

[19] G.M. Barratt, Therapeutic applications of colloidal drug carriers, Pharm. Sci. Technol. Today 3 (2000) 163-171.

[20] C. Vauthier, P. Couvreur, Nanomedicines: a new approach for the treatment of serious diseases, J. Biomed. Nanotechnol. 3 (2007) 223-234.

[21] M.L. Forrest, G.S. Kwon, Clinical developments in drug delivery nanotechnology, Adv. Drug Deliv. Rev. 60 (2008) 861-862.

[22] M.K. Sharma, V.K. Rao, G.S. Agarwal, G.P. Rai, N. Gopalan, S. Prakash, S.K. Sharma, R. Vijayaraghavan, Highly sensitive amperometric immunosensor for detection of Plasmodium falciparum histidine-rich protein 2 in serum of humans with malaria: comparison with a commercial kit, J. Clin. Microbiol. 46 (2008) 3759-3765.

[23] V. Wiwanitkit, Alternative tools for field analysis on malarial infection: a reappraisal, Clin. Ther. 160 (2009) 83-85.

[24] O. Kayser, A.F. Kiderlen, Delivery strategies for antiparasitics, Expert Opin. Invest. Drugs 12 (2003) 197-207.

[25] A.A. Date, M.D. Joshi, V.B. Patravale, Parasitic diseases: Liposomes and polymeric nanoparticles versus lipid nanoparticles, Adv. Drug Deliv. Rev. 59 (2007) 505-521.

[26] C.R. Alving, Design and selection of vaccine adjuvants: animal models and human trials, Vaccine 20 (2002) 856-864.

[27] K.E. Kester, D.A. McKinney, N. Tornieporth, C.F. Ockenhouse, D.G. Heppner Jr., T. Hall, B.T. Wellde, K. White, P. Sun, R. Schwenk, U. Krzych, M. Delchambre, G. Voss, M.C. Dubois, R.A. Gasser Jr., M.G. Dowler, M. O'Brien, J. Wittes, R. Wirtz, J. Cohen, W.R. Ballou, A phase I/Ila safety, immunogenicity, and efficacy bridging randomized study of a two-dose regimen of liquid and lyophilized formulations of the candidate malaria vaccine RTS, S/AS02A in malaria-naive adults, Vaccine 25 (2007) 5359-5366.

[28] B. Combadière, B. Mahe, Particle-based vaccines for transcutaneous vaccination, Comp. Immunol. Microbiol. Infect. Dis. 31 (2008) 293-315.

[29] L.J. Peek, C.R. Middaugh, C. Berkland, Nanotechnology in vaccine delivery, Adv. Drug Deliv. Rev. 60 (2008) 915-928.

[30] P.N. Newton, S. Ward, B.J. Angus, W. Chierakul, A. Dondorp, R. Ruangveerayuth, K. Silamut, P. Teerapong, Y. Suputtamongkol, S. Looareesuwan, N.J. White, Early treatment failure in severe malaria resulting from abnormally low plasma quinine concentrations, Trans. R. Soc. Trop. Med. Hyg. 100 (2006) 184-186.

[31] A. Trouet, P. Pirson, R. Steiger, Development of new derivatives of primaquine by association with lysosomotropic carriers, Bull. World Health Organ. 59 (1981) 449-458.

[32] V.C.F. Mosqueira, P.M. Loiseau, C. Bories, P. Legrand, J.P. Devissaguet, G. Barratt, Efficacy and pharmacokinetics of intravenous nanocapsule formulations of halofantrine in Plasmodium berghei-infected mice, Antimicrob. Agents Chemother. 48 (2004) 1222-1228.

[33] E.A. Leite, A. Grabe-Guimarães, H.N. Guimarães, G.L.L. Machado-Coelho, G. Barratt, V.C.F. Mosqueira, Cardiotoxicity reduction induced by halofantrine entrapped in nanocapsule devices, Life Sci. 80 (2007) 1327-1334.

[34] A.M. Carcaboso, R.M. Hernández, M. Igartua, A.R. Gascón, J.E Rosas, M.E. Patarroyo, J.L. Pedraz, Immune response after oral administration of the encapsulated malaria synthetic peptide SPf66, Int. J. Pharm. 260 (2003) 273-282.

[35] A.U. Krettli, L.H. Miller, Malaria: A sporozoite runs through it, Curr. Biol. 11 (2001) R409-R412.

[36] WHO, Guidelines for the Treatment of Malaria (2006), http://www.whqlibdoc. who.int/publications/2006/9241546948_eng.pdf, accessed on 20th March 2009.

[37] S. Mohanty, D.K. Patel, S.S. Pati, S.K. Mishra, Adjuvant therapy in cerebral malaria, Ind. J. Med. Res. 124 (2006) 245-260.

[38] N. Coltel, V. Combes, N.H. Hunt, G.E. Grau, Cerebral malaria - a neurovascular pathology with many riddles still to be solved, Curr. Neurovasc. Res. 1 (2004) 91-110.

[39] B. Pouvelle, V. Matarazzo, C. Jurzynski, J. Nemeth, M. Ramharter, G. Rougon, J. Gysin, Neural cell adhesion molecule, a new cytoadhesion receptor for Plasmodium falciparum-infected erythrocytes capable of aggregation, Infect. Immun. 75 (2007) 3516-3522

[40] C.J.M. Whitty, F. Sanderson, New therapies and changing patterns of treatment for malaria, Curr. Opin. Infect. Dis. 12 (1999) 579-584

[41] WHO, Susceptibility of Plasmodium falciparum to antimalarial drugs: report on global monitoring (2005), www.who.int/malaria/rbm/Attachment/20041108/ SusceptibilityPlasmodium_report.pdf, accessed on 25th March 2009.

[42] R.N. Price, G. Dorsey, E.A. Ashley, K.I. Barnes, J.K. Baird, U. d'Alessandro, P.J. Guerin, M.K. Laufer, I. Naidoo, F. Nosten, P. Olliaro, C.V. Plowe, P. Ringwald, C.H. 
Sibley, K. Stepniewska, N.J. White, World antimalarial resistance network I: Clinical efficacy of antimalarial drugs, Malaria J. 6 (2007) 1-9.

[43] K. Becker, K. Kirk, Of malaria, metabolism and membrane transport, Trends Parasitol. 20 (2004) 590-596.

[44] S.G. Valderramos, D.A. Fidock, Transporters involved in resistance to antimalarial drugs, Trends Pharmacol. Sci. 27 (2006) 594-601.

[45] D.A. Fidock, R.T. Eastman, S.A. Ward, S.R. Meshnick, Recent highlights in antimalarial drug resistance and chemotherapy research, Trends Parasitol. 24 (2008) 537-544.

[46] N. Vale, R. Moreira, P. Gomes, Primaquine revisited six decades after its discovery, Eur. J. Med. Chem. 44 (2009) 937-953.

[47] M. Crockett, K.C. Kain, Tafenoquine: a promising new antimalarial agent, Expert Opin. Investig. Drugs 16 (2007) 705-715.

[48] G.A. Biagini, S.A. Ward, P.G. Bray, Malaria parasite transporters as a drug-delivery strategy, Trends Parasitol. 21 (2005) 299-301.

[49] A.M. Lehane, R. Hayward, K.J. Saliba, K. Kirk, A verapamil-sensitive chloroquineassociated $\mathrm{H}^{+}$leak from the digestive vacuole in chloroquine-resistant malaria parasites, J. Cell Sci. 121 (2008) 1624-1632.

[50] L.M. Bareford, P.W. Swaan, Endocytic mechanisms for targeted drug delivery, Adv. Drug Deliv. Rev. 59 (2007) 748-758.

[51] L.W. Ho, R. Bendayan, A.M. Rauth, Y.X. Hui, K. Babakhanian, Y.W. Xiao, A mechanistic study of enhanced doxorubicin uptake and retention in multidrug resistant breast cancer cells using a polymer-lipid hybrid nanoparticle system, J. Pharmacol. Exp. Ther. 317 (2006) 1372-1381.

[52] L. Qiu, N. Jing, Y. Jin, Preparation and in vitro evaluation of liposomal chloroquine diphosphate loaded by a transmembrane pH-gradient method, Int. J. Pharm. 361 (2008) 56-63.

[53] C.R.J.C. Newton, S. Krishna, Severe falciparum malaria in children: Current understanding of pathophysiology and supportive treatment, Pharmacol. Ther. 79 (1998) $1-53$.

[54] S.K. Mishra, C.R. Newton, Diagnosis and management of the neurological complications of falciparum malaria, Nat. Rev. Neurol. 5 (2009) 189-198.

[55] S. Krishna, L. Bustamante, R.K. Haynes, H.M. Staines, Artemisinins: their growing importance in medicine, Trends Pharmacol. Sci. 29 (2008) 520-527.

[56] B. Pouvelle, R. Spiegel, L. Hsiao, R.J. Howard, R.L. Morris, A.P. Thomas, T.F. Taraschi, Direct access to serum macromolecules by intraerythrocytic malaria parasites, Nature 353 (1991) 73-75.

[57] I.D. Goodyer, B. Pouvelle, T.G. Schneider, D.P. Trelka, T.F. Taraschi, Characterization of macromolecular transport pathways in malaria-infected erythrocytes, Mol. Biochem. Parasitol. 87 (1997) 13-28.

[58] S. Charpian, J.M. Przyborski, Protein transport across the parasitophorous vacuole of Plasmodium falciparum: Into the great wide open, Traffic 9 (2008) 157-165.

[59] H.J. Vial, S. Wein, C. Farenc, C. Kocken, O. Nicolas, M.L. Ancelin, F. Bressolle, A. Thomas, M. Calas, Prodrugs of bisthiazolium salts are orally potent antimalarials, Proc. Natl. Acad. Sci. U. S. A. 101 (2004) 15458-15463.

[60] E. Richier, G.A. Biagini, S. Wein, F. Boudou, P.G. Bray, S.A. Ward, E. Precigout, M. Calas, J.F. Dubremetz, H.J. Vial, Potent antihematozoan activity of novel bisthiazolium drug T16: Evidence for inhibition of phosphatidylcholine metabolism in erythrocytes infected with Babesia and Plasmodium spp, Antimicrob. Agents Chemother. 50 (2006) 3381-3388.

[61] P. Gornicki, Apicoplast fatty acid biosynthesis as a target for medical intervention in Apicomplexan parasites, Int. J. Parasitol. 33 (2003) 885-896,

[62] G.A. Biagini, E.M. Pasini, R. Hughes, H.P. De Koning, H.J. Vial, P.M. O'Neill, S.A. Ward, P.G. Bray, Characterization of the choline carrier of Plasmodium falciparum: a route for the selective delivery of novel antimalarial drugs, Blood 104 (2004) 3372-3377.

[63] G. Barratt, Colloidal drug carriers: Achievements and perspectives, Cell. Mol. Life Sci. 60 (2003) 21-37.

[64] H. Devalapally, A. Chakilam, M.M. Amiji, Role of nanotechnology in pharmaceutical product development, J. Pharm. Sci. 96 (2007) 2547-2565.

[65] J. Wong, A. Brugger, A. Khare, M. Chaubal, P. Papadopoulos, B. Rabinow, J. Kipp, J. Ning, Suspensions for intravenous (IV) injection: a review of development, preclinical and clinical aspects, Adv. Deliv. Rev. 60 (2008) 939-954.

[66] D.E. Owens Iii, N.A. Peppas, Opsonization, biodistribution, and pharmacokinetics of polymeric nanoparticles, Int. J. Pharm. 307 (2006) 93-102.

[67] P. Legrand, V. Mosqueira, P. Loiseau, C. Bories, G. Barratt, Long circulating nanocapsules: Interest in the treatment of severe malaria with halofantrine, Ann. Pharm. Fr. 61 (2003) 196-202.

[68] B. Chimanuka, M. Gabriëls, M.R. Detaevernier, J.A. Plaizier-Vercammen, Preparation of beta-artemether liposomes, their HPLC-UV evaluation and relevance for clearing recrudescent parasitaemia in Plasmodium chabaudi malaria-infected mice, J. Pharm. Biomed. 28 (2002) 13-22.

[69] K.J. Longmuir, R.T. Robertson, S.M. Haynes, J.L. Baratta, A.J. Waring, Effective targeting of liposomes to liver and hepatocytes in vivo by incorporation of a Plasmodium amino acid sequence, Pharm. Res. 23 (2006) 759-769.

[70] M. Owais, G.C. Varshney, A. Choudhury, S. Chandra, C.M. Gupta, Chloroquine encapsulated in malaria-infected erythrocyte-specific antibody-bearing liposomes effectively controls chloroquine-resistant Plasmodium berghei infections in mice, Antimicrob. Agents Chemother. 39 (1995) 180-184.

[71] Y. Gupta, A. Jain, S.K. Jain, Transferrin-conjugated solid lipid nanoparticles for enhanced delivery of quinine dihydrochloride to the brain, J. Pharm. Pharmacol. 59 (2007) 935-940.

[72] F. Föger, W. Noonpakdee, B. Loretz, S. Joojuntr, W. Salvenmoser, M. Thaler, A. Bernkop-Schnürch, Inhibition of malarial topoisomerase II in Plasmodium falciparum by antisense nanoparticles, Int. J. Pharm. 319 (2006) 139-146.
[73] S.E. Haas, C.C. Bettoni, L.K. de Oliveira, S.S. Guterres, T. Dalla Costa, Nanoencapsulation increases quinine antimalarial efficacy against Plasmodium berghei in vivo, Int.J. Antimicrob. Agents 34 (2009) 156-161.

[74] D.D. Lasic, F.J. Martin, E. Mayhew, Stealth liposomes, Ann. Biomed. Eng. 19 (1991) 594.

[75] R. Gref, A. Domb, P. Quellec, T. Blunk, R.H. Muller, J.M. Verbavatz, R. Langer, The controlled intravenous delivery of drugs using PEG-coated sterically stabilized nanospheres, Adv. Drug Deliv. Rev. 16 (1995) 215-233.

[76] M.C. Garnett, Targeted drug conjugates: Principles and progress, Adv. Drug Deliv. Rev. 53 (2001) 171-216.

[77] G.L. Scherphof, M. Velinova, J. Kamps, J. Donga, H. Van der Want, F. Kuipers, L. Havekes, T. Daemen, Modulation of pharmacokinetic behavior of liposomes, Adv. Drug Deliv. Rev. 24 (1997) 179-191.

[78] J.M. Rodrigues, H. Fessi, C. Bories, F. Puisieux, J.P. Devissaguet, Primaquine-loaded poly(lactide) nanoparticles: Physicochemical study and acute tolerance in mice, Int. J. Pharm. 126 (1995) 253-260.

[79] B. Heurtault, P. Legrand, V. Mosqueira, J.P. Devissaguet, G. Barratt, C. Bories, The antileishmanial properties of surface-modified, primaquine-loaded nanocapsules tested against intramacrophagic Leishmania donovani amastigotes in vitro, Ann. Trop. Med. Parasitol. 95 (2001) 529-533.

[80] M. Lück, B.R. Paulke, W. Schröder, T. Blunk, R.H. Müller, Analysis of plasma protein adsorption on polymeric nanoparticles with different surface characteristics, J. Biomed. Mater. Res. 39 (1998) 478-485.

[81] V.P. Torchilin, Multifunctional nanocarriers, Adv. Drug Deliv. Rev. 58 (2006) $1532-1555$.

[82] V.C.F. Mosqueira, P. Legrand, A. Gulik, O. Bourdon, R. Gref, D. Labarre, G. Barratt, Relationship between complement activation, cellular uptake and surface physicochemical aspects of novel PEG-modified nanocapsules, Biomaterials 22 (2001) 2967-2979.

[83] V.C.F. Mosqueira, P. Legrand, J.L. Morgat, M. Vert, E. Mysiakine, R. Gref, J.P Devissaguet, G. Barratt, Biodistribution of long-circulating PEG-grafted nanocapsules in mice: Effects of PEG chain length and density, Pharm. Res. 18 (2001) 1411-1419.

[84] V.C. Mosqueira, P. Legrand, G. Barratt, Surface-modified and conventional nanocapsules as novel formulations for parenteral delivery of halofantrine, J. Nanosci. Nanotechnol. 6 (2006) 3193-3202.

[85] S.M. Haynes, K.J. Longmuir, R.T. Robertson, J.L. Baratta, A.J. Waring, Liposomal polyethyleneglycol and polyethyleneglycol-peptide combinations for active targeting to liver in vivo, Drug Deliv. 15 (2008) 207-217.

[86] A. Singhal, C.M. Gupta, Antibody-mediated targeting of liposomes to red cells in vivo, FEBS Lett. 201 (1986) 321-326.

[87] A.K. Agrawal, A. Singhal, C.M. Gupta, Functional drug targeting to erythrocytes in vivo using antibody bearing liposomes as drug vehicles, Biochem. Biophys. Res. Commun. 148 (1987) 357-361.

[88] S. Chandra, A.K. Agrawal, C.M. Gupta, Chloroquine delivery to erythrocytes in Plasmodium berghei-infected mice using antibody-bearing liposomes as drug vehicles, J. Biosci. 16 (1991) 137-144.

[89] P.A.M. Peeters, B.G. Brunink, W.M.C. Eling, D.J.A. Crommelin, Therapeutic effect of chloroquine (CQ)-containing immunoliposomes in rats infected with Plasmodium berghei parasitized mouse red blood cells: Comparison with combinations of antibodies and CQ or liposomal CQ, BBA - Biomembranes 981 (1989) 269-276.

[90] A.K. Agrawal, C.M. Gupta, Tuftsin-bearing liposomes in treatment of macrophagebased infections, Adv. Drug Deliv. Rev. 41 (2000) 135-146.

[91] R.T. Robertson, J.L. Baratta, S.M. Haynes, K.J. Longmuir, Liposomes incorporating a Plasmodium amino acid sequence target heparan sulfate binding sites in liver, J. Pharm. Sci. 97 (2008) 3257-3273.

[92] A.D. Bangham, M.M. Standish, J.C. Watkins, Diffusion of univalent ions across the lamellae of swollen phospholipids. J. Mol. Biol. 13 (1965) 238-252.

[93] G. Gregoriadis, P.D. Leathwood, B.E. Ryman, Enzyme entrapment in liposomes, FEBS Lett. 14 (1971) 95-99.

[94] G. Gregoriadis, Drug entrapment in liposomes: possibilities for chemotherapy, Biochem. Soc. Trans. 2 (1974) 117-119.

[95] E. Sponton, D. Drouin, J. Delattre, Design of sterile muramyldipeptide-containing oligolamellar liposomes suitable for i.v. injection. Effect of liposome size and lipid composition on their ability to render peritoneal macrophages antitumoral, Int. J. Pharm. 23 (1985) 299-313.

[96] D.D. Lasic, Novel applications of liposomes, Trends Biotechnol. 16 (1998) 307-321.

[97] G.A. Ramaldes, J.R. Deverre, J.M. Grognet, F. Puisieux, E. Fattal, Use of an enzyme immunoassay for the evaluation of entrapment efficiency and in vitro stability in intestinal fluids of liposomal bovine serum albumin, Int. J. Pharm. 143 (1996) $1-11$.

[98] C. Weingarten, A. Moufti, J.F. Desjeux, Oral ingestion of insulin liposomes: Effects of the administration route, Life Sci. 28 (1981) 2747-2752.

[99] A. Laham, N. Claperon, J.J. Durussel, E. Fattal, J. Delattre, F. Puisieux, P. Couvreur, P. Rossignol, Intracarotidal administration of liposomally-entrapped ATP: Improved efficiency against experimental brain ischemia, Pharmacol. Res. Commun. 20 (1988) 699-705.

[100] A.C. Allison, G. Gregoriadis, Liposomes as immunological adjuvants, Nature 252 (1974) 252

[101] C.R. Alving, R.L. Richards, Liposomes containing lipid A: A potent nontoxic adjuvant for a human malaria sporozoite vaccine, Immunol. Lett. 25 (1990) 275-279.

[102] D. Papahadjopoulos, A. Gabizon, Liposomes designed to avoid the reticuloendothelial system, Prog. Clin. Biol. Res. 343 (1990) 85-93.

[103] V.P. Torchilin, Recent advances with liposomes as pharmaceutical carriers, Nat Rev. Drug Discov. 4 (2005) 145-160.

104] P. Pirson, R.F. Steiger, A. Trouet, Liposomes in the chemotherapy of experimental murine malaria, Trans. R. Soc. S. Afr. 73 (1979) 347. 
[105] J.E. Smith, P. Pirson, R.E. Sinden, Studies on the kinetics of uptake and distribution of free and liposome-entrapped primaquine, and of sporozoites by isolated perfused rat liver, Ann. Trop. Med. Parasitol. 77 (1983) 379-386.

[106] T.D. Madden, P.R. Harrington, L.C.L. Tai, M.B. Bally, L.D. Mayer, T.E. Redelmeier, H.C. Loughrey, C.P.S. Tilcock, L.W. Reinish, P.R. Cullis, The accumulation of drugs within large unilamellar vesicles exhibiting a proton gradient: a survey, Chem. Phys. Lipids 53 (1990) 37-46.

[107] P.R. Cullis, M.J. Hope, M.B. Bally, T.D. Madden, L.D. Mayer, D.B. Fenske, Influence of $\mathrm{pH}$ gradients on the transbilayer transport of drugs, lipids, peptides and metal ions into large unilamellar vesicles, BBA - Rev. Biomembranes 1331 (1997) 187-211.

[108] A. Brossi, B. Venugopalan, L. Dominguez Gerpe, H.J.C. Yeh, J.L. Flippen-Anderson, P. Buchs, X.D. Luo, W. Milhous, W. Peters, Arteether, a new antimalarial drug: Synthesis and antimalarial properties, J. Med. Chem. 31 (1988) 645-650.

109] A.A. Al-Angary, M.A. Al-Meshal, M.A. Bayomi, S.H. Khidr, Evaluation of liposoma formulations containing the antimalarial agent, arteether, Int. J. Pharm. 128 (1996) 163-168.

[110] M.A. Bayomi, A.A. Al-Angary, M.A. Al-Meshal, M.M. Al-Dardiri, In vivo evaluation of arteether liposomes, Int. J. Pharm. 175 (1998) 1-7.

[111] M. Gabriels, J. Plaizier-Vercammen, Physical and chemical evaluation of liposomes, containing artesunate, J. Pharm. Biomed. Anal. 31 (2003) 655-667.

[112] P. Pirson, R.F. Steiger, A. Trouet, Primaquine liposomes in the chemotherapy of experimental murine malaria, Ann. Trop. Med. Parasitol. 74 (1980) 383-391.

[113] P. Pirson, R. Steiger, A. Trouet, The disposition of free and liposomally encapsulated antimalarial primaquine in mice, Biochem. Pharmacol. 31 (1982) 3501-3507.

[114] P.A.M. Peeters, K. De Leest, W.M.C. Eling, D.J.A. Crommelin, Chloroquine blood levels after administration of the liposome-encapsulated drug in relation to therapy of murine malaria, Pharm. Res. 6 (1989) 787-793.

[115] B. Arica, A.Y. Ozer, M.T. Ercan, A.A. Hincal, Characterization, in vitro and in vivo studies on primaquine diphosphate liposomes, J. Microencapsul 12 (1995) 469-485.

[116] N.S. Postma, C.C. Hermsen, J. Zuidema, W.M.C. Eling, Plasmodium vinckei: Optimization of desferrioxamine B delivery in the treatment of murine malaria, Exp. Parasitol. 89 (1998) 323-330.

117] G. Stensrud, S.A. Sande, S. Kristensen, G. Smistad, Formulation and characterisation of primaquine loaded liposomes prepared by a $\mathrm{pH}$ gradient using experimental design, Int. J. Pharm. 198 (2000) 213-228.

[118] I. Henriksen, S.R. Vågen, S.A. Sande, G. Smistad, J. Karlsen, Interactions between liposomes and chitosan II: Effect of selected parameters on aggregation and leakage, Int. J. Pharm. 146 (1997) 193-203.

[119] C.R. Alving, Design and selection of vaccine adjuvants: animal models and human trials, Vaccine 20 (Suppl. 3) (2002) S56-S64.

[120] R.K. Tyagi, P.K. Sharma, S.P. Vyas, A. Mehta, Various carrier system(s) mediated genetic vaccination strategies against malaria, Expert Rev. Vaccines 7 (2008) 499-520.

[121] R.L. Richards, M.D. Hayre, W.T. Hockmeyer, C.R. Alving, Liposomes, lipid A, and aluminium hydroxide enhance the immune response to a synthetic malaria sporozoite antigen, Infect. Immun. 56 (1988) 682-686.

[122] R.L. Richards, M. Rao, N.M. Wassef, G.M. Glenn, S.W. Rothwell, C.R. Alving Liposomes containing lipid A serve as an adjuvant for induction of antibody and cytotoxic T-cell responses against RTS, S malaria antigen, Infect. Immun. 66 (1998) 2859-2865.

[123] J.N. Verma, M. Rao, S. Amselem, U. Krzych, C.R. Alving, S.J. Green, N.M. Wassef Adjuvant effects of liposomes containing lipid A: Enhancement of liposomal antigen presentation and recruitment of macrophages, Infect. Immun. 60 (1992) $2438-2444$

[124] S.K. Sharma, C. Gupta, V. Dwivedi, S. Misra-Bhattacharya, O. Mohammad, Prophylactic potential of liposomized integral membrane protein of Plasmodium yoelii nigeriensis against blood stage infection in BALB/c mice, Vaccine 25 (2007) 2103-2111.

[125] M. Joshi, S. Pathak, S. Sharma, V. Patravale, Design and in vivo pharmacodynamic evaluation of nanostructured lipid carriers for parenteral delivery of artemether: Nanoject, Int. J. Pharm. 364 (2008) 119-126.

[126] S.A. Wissing, O. Kayser, R.H. Muller, Solid lipid nanoparticles for parenteral drug delivery, Adv. Drug Deliv. Rev. 56 (2004) 1257-1272.

[127] A.M. Dierling, Z. Cui, Targeting primaquine into liver using chylomicron emulsions for potential vivax malaria therapy, Int. J. Pharm. 303 (2005) 143-152.
[128] K.K. Singh, S.K. Vingkar, Formulation, antimalarial activity and biodistribution of oral lipid nanoemulsion of primaquine, Int. J. Pharm. 347 (2008) 136-143.

[129] S.D. Mandawgade, S. Sharma, S. Pathak, V.B. Patravale, Development of SMEDDS using natural lipophile: Application to beta-Artemether delivery, Int. J. Pharm. 362 (2008) 179-183.

[130] M. Joshi, S. Pathak, S. Sharma, V. Patravale, Solid microemulsion preconcentrate (NanOsorb) of artemether for effective treatment of malaria, Int. J. Pharm. 362 (2008) 172-178.

[131] T. Xu, N. Zhang, H.L. Nichols, D. Shi, X. Wen, Modification of nanostructured materials for biomedical applications, Mater. Sci. Eng. C 27 (2007) 579-594.

[132] V.D. Labhasetwar, A.K. Dorle, Nanoparticles - A colloidal drug delivery system for primaquine and metronidazole, J. Control. Release 12 (1990) 113-119.

[133] D. Bhadra, S. Bhadra, N.K. Jain, PEGylated peptide-based dendritic nanoparticulate systems for delivery of artemether, J. Drug Deliv. Sci. Technol. 15 (2005) 65-73.

[134] D. Bhadra, S. Bhadra, N.K. Jain, PEGylated peptide dendrimeric carriers for the delivery of antimalarial drug chloroquine phosphate, Pharm. Res. 23 (2006) 623-633.

[135] D. Bhadra, A.K. Yadav, S. Bhadra, N.K. Jain, Glycodendrimeric nanoparticulate carriers of primaquine phosphate for liver targeting, Int. J. Pharm. 295 (2005) 221-233.

[136] M.E. Brewster, T. Loftsson, Cyclodextrins as pharmaceutical solubilizers, Adv. Drug Deliv. Rev. 59 (2007) 645-666.

[137] J.W. Wong, K.H. Yuen, Improved oral bioavailability of artemisinin through inclusion complexation with beta- and gama-cyclodextrins, Int. J. Pharm. 227 (2001) 177-185.

[138] I.E. Crandall, W.A. Szarek, J.Z. Vlahakis, Y. Xu, R. Vohra, J. Sui, R. Kisilevsky, Sulfated cyclodextrins inhibit the entry of Plasmodium into red blood cells. Implications for malarial therapy, Biochem. Pharmacol. 73 (2007) 632-642.

[139] M.G. Hartell, R. Hicks, A.K. Bhattacharjee, B.W. Koser, K. Carvalho, J.E. Van Hamont, Nuclear magnetic resonance and molecular modeling analysis of the interaction of the antimalarial drugs artelinic acid and artesunic acid with betacyclodextrin, J. Pharm. Sci. 93 (2004) 2076-2089.

[140] C.O. Onyeji, S.I. Omoruyi, F.A. Oladimeji, Dissolution properties and characterization of halofantrine-2-hydroxypropyl-beta-cyclodextrin binary systems, Pharmazie 62 (2007) 858-863.

[141] M.T. Ansari, I. Iqbal, V.B. Sunderland, Dihydroartemisinin-cyclodextrin complexation: Solubility and stability, Arch. Pharm. Res. 32 (2009) 155-165.

[142] R.H. Müller, S. Gohla, A. Dingler, T. Schneppe, Large scale production of solid lipid nanoparticles (SLN ${ }^{\mathrm{TM}}$ ) and nanosuspensions (DissoCubes ${ }^{\mathrm{TM}}$ ), in: D. Wise (Ed.), Handbook of Pharmaceutical Controlled Release Technology, Marcel Dekker, New York, 2000, pp. 359-376.

[143] V.B. Patravale, A.A. Date, R.M. Kulkarni, Nanosuspensions: a promising drug delivery strategy, J. Pharm. Pharmacol. 56 (2004) 827-840.

[144] J. Chingunpitak, S. Puttipipatkhachorn, P. Chavalitshewinkoon-Petmitr, Y. Tozuka, K. Moribe, K. Yamamoto, Formation, physical stability and in vitro antimalarial activity of dihydroartemisinin nanosuspensions obtained by cogrinding method, Drug Dev. Ind. Pharm. 34 (2008) 314-322.

[145] H. Fessi, F. Piusieux, J.P. Devissaguet, N. Ammoury, S. Benita, Nanocapsule formation by interfacial polymer deposition following solvent displacement, Int. J. Pharm. 55 (1989).

[146] P. Couvreur, G. Barratt, E. Fattal, P. Legrand, C. Vauthier, Nanocapsule technology: A review, Crit. Rev. Ther. Drug 19 (2002) 99-134.

[147] N.P. da Silva Santos, S.C. Nascimento, M.S.O. Wanderley, N.T. Pontes-Filho, J.F. da Silva, C.M.M.B. de Castro, E.C. Pereira, N.H. da Silva, N.K. Honda, N.S. SantosMagalhães, Nanoencapsulation of usnic acid: An attempt to improve antitumour activity and reduce hepatotoxicity, Eur. J. Pharm. Biopharm. 64 (2006) 154-160.

[148] P. Legrand, G. Barratt, V. Mosqueira, H. Fessi, J.P. Devissaguet, Polymeric nanocapsules as drug delivery systems: a review, S. T. P. Pharm. Sci. 9 (1999) 411-418.

[149] H.C. Pedroni, C.C. Bettoni, S.M. Spalding, T. Dalla Costa, Plasmodium berghei: Development of an irreversible experimental malaria model in Wistar rats, Exp. Parasitol. 113 (2006) 193-196.

[150] F. Maestrelli, P. Mura, M.J. Alonso, Formulation and characterization of triclosan sub-micron emulsions and nanocapsules, J. Microencapsul 21 (2004) 857-864.

[151] WHO, Global Health Observatory Map Gallery (2008), http://gamapserver.who. int/mapLibrary/Files/Maps/Global_Malaria_ITHRiskMap.JPG, acessed on 30th June 2009. 